DRAFT VERSION MARCH 6, 2018

Preprint typeset using LTEX style emulateapj v. 5/2/11

\title{
GALAXY GROUPS IN THE 2MASS REDSHIFT SURVEY
}

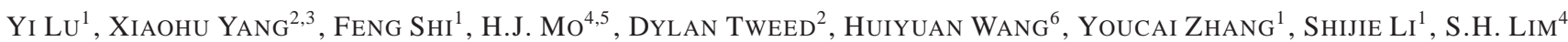 \\ Draft version March 6, 2018
}

\begin{abstract}
A galaxy group catalog is constructed from the 2MASS Redshift Survey (2MRS) with the use of a halobased group finder. The halo mass associated with a group is estimated using a 'GAP' method based on the luminosity of the central galaxy and its gap with other member galaxies. Tests using mock samples shows that this method is reliable, particularly for poor systems containing only a few members. On average $80 \%$ of all the groups have completeness $>0.8$, and about $65 \%$ of the groups have zero contamination. Halo masses are estimated with a typical uncertainty $\sim 0.35$ dex. The application of the group finder to the 2MRS gives 29,904 groups from a total of 43,246 galaxies at $z \leq 0.08$, with 5,286 groups having two or more members. Some basic properties of this group catalog is presented, and comparisons are made with other groups catalogs in overlap regions. With a depth to $z \sim 0.08$ and uniformly covering about $91 \%$ of the whole sky, this group catalog provides a useful data base to study galaxies in the local cosmic web, and to reconstruct the mass distribution in the local Universe.
\end{abstract}

Subject headings: large-scale structure of universe - dark matter - galaxies: halos - methods: statistical

\section{INTRODUCTION}

One important goal in modern cosmology is to establish the relationship between galaxies and dark matter halos in which galaxies form and reside. Understanding this galaxy-halo connection can provide important information about the underlying processes governing galaxy formation and evolution. Theoretically, there are several ways to study this relationship. The first is to use numerical simulations (Springel et al. 2005; Wadsley et al. 2004; Bryan et al. 1995; Kravtsov et al. 2002; Teyssier 2002; Springel 2010) or semianalytical models (van den Bosch 2002; Kang et al. 2005; Croton et al. 2006). These approaches incorporate various physical processes that are potentially important for galaxy formation and evolution, such as gas cooling, star formation, feedback mechanisms, and so on. However, many processes in such modeling have to be approximated by subgrid implementations and simple parameterizations, and so the results obtained are still questionable and sometimes fail to match observations. An alternative method to establish the galaxy-dark matter halo connection is to adopt an empirical approach. Models in this category includes the halo occupation model (e.g. Jing et al. 1998; Peacock et al. 2000; Berlind \& Weinberg 2002; Zheng et al. 2005), the conditional luminosity functions (e.g. Yang, Mo \& van den Bosch 2003; van den Bosch, Yang \& Mo 2003; Yan, Madgwick \& White 2003; Tinker 2005; Zheng et al. 2005; Cooray et al. 2006; van den Bosch et al. 2007; Yang et al. 2012), halo abundance matching (e.g. Mo et al. 1999; Vale \& Ostriker 2004;

\footnotetext{
${ }^{1}$ Key Laboratory for Research in Galaxies and Cosmology, Shanghai Astronomical Observatory, Nandan Road 80, Shanghai 200030, China; Email: luyi@shao.ac.cn

${ }^{2}$ Center for Astronomy and Astrophysics, Shanghai Jiao Tong University, Shanghai 200240, China; E-mail: xyang@ @jtu.edu.cn

${ }^{3}$ IFSA Collaborative Innovation Center, Shanghai Jiao Tong University, Shanghai 200240, China

${ }^{4}$ Department of Astronomy, University of Massachusetts, Amherst MA 01003-9305, USA

${ }^{5}$ Physics Department and Center for Astrophysics, Tsinghua University, Beijing 10084, China

${ }^{6}$ Key Laboratory for Research in Galaxies and Cosmology, Department of Astronomy, University of Science and Technology of China, Hefei, Anhui 230026, China
}

Conroy et al. 2006; Behroozi et al. 2010; Guo et al. 2010; Trujillo-Gomez et al. 2011), and parametric model fitting (Lu et al. 2014, 2015). By construction, the empirical approach can produce much better fits to the observational data than numerical simulations and semi-analytical models, and so the galaxy-halo relationship established in this way is more accurate. Yet another way of to establish the galaxy-dark matter halo connection is to identify galaxy systems (clusters and groups, collectively referred to as groups in the following) to represent dark halos. With a well-defined galaxy group catalog, one can not only study the relationship between halos and galaxies (e.g. Yang et al. 2005a, 2008; Lan et al. 2016; Erfanianfar et al. 2014; Rodríguez-Puebla et al. 2015; Jiang et al. 2016), but also investigate how dark matter halos trace the large-scale structure of the universe (e.g. Yang et al. 2005b; Yang et al. 2005c; Tal et al. 2014). In addition to these statistical studies, a well-defined group sample can also be used to reconstruct the current and initial cosmic density fields, so as to study not only the structures but also the formation histories of the cosmic web (e.g. Wang et al. 2012, 2013, 2014).

The quality of a group sample depends on the group finder used to identify individual groups. During the past two decades, numerous group catalogs have been constructed from various observations, including the 2-degree Field Galaxy Redshift Survey (2dFGRS) (Eke et al. 2004; Yang et al. 2005a), the DEEP2 survey (Crook et al. 2007) and the Sloan Digital Sky Survey (SDSS) (e.g. Berlind et al. 2006; Yang et al. 2007; Tago et al. 2010; Nurmi et al. 2013). The group finders adopted in these investigations range from the traditional friends-of-friends (FOF) algorithm (e.g. Davis et al. 1985), to the hybrid matched filter method (Kim et al. 2002) and the "MaxBCG" method (Koester et al. 2007). Although the accuracy of a particular group finder depends on the properties of the observational sample, all group finders need to handle the same observational effects, such as redshift distortion that impacts the clustering pattern of galaxies, and the variations of the mean inter-galaxy separation due to apparent magnitude limit.

In this paper, we present our construction of a galaxy group 
catalog from the 2MASS Redshift Survey (2MRS), which is complete roughly to $K_{s}=11.75$ and covers $91 \%$ of the sky (Huchra et al. 2012). Several group catalogs have already been constructed from 2MRS. Crook et al. (2007) constructed a group catalog using galaxies with a magnitude limit at $K_{s}=$ 11.25 and a FOF algorithm similar to that of Huchra \& Geller (1982). Tully (2015) built a group catalog in the volume between 3,000 and $10,000 \mathrm{~km} \mathrm{~s}^{-1}$ using a methodology similar to that of Yang et al. (2005a). Tempel et al. (2016) constructed a group catalog to larger distances using a FOF algorithm. Our goal here is to obtain a reliable and uniform galaxy group catalog using all galaxies in the $2 \mathrm{MRS}$ brighter than $K_{s}=11.75$ to a redshift $z=0.08$. By involving a new halo mass estimation method, we are trying to obtain a better representive halo distributions in the local Universe.

The group finder to be used is the halo-based group finder developed by Yang et al. (2005a), which groups galaxies within their host dark matter halos. This group finder is suitable to study the relation between galaxies and dark matter haloes over a wide range of halo masses, from rich clusters of galaxies to poor galaxy groups. It has been tested with mock galaxy surveys, and has been applied quite successfully to several galaxy catalogs (Yang et al. 2005a; Weinmann et al. 2006a; Yang et al. 2007). The essential idea behind this group finder is to use the relationships between halo mass and its size and velocity dispersion when deciding the membership of a group. Thus an accurate estimate of the halo mass for a candidate galaxy group is a key step. As shown in Yang et al. (2007), for relative deep surveys, such as the SDSS, the group total luminosity (or stellar mass) provides a reliable ranking of the halo mass. In this case, halo masses can be estimated reliably by matching the rank of the characteristic luminosity of a group to that of halo mass given by a halo mass function. However, as pointed out in Lu et al. (2015), for a shallow survey, such as the $2 \mathrm{MRS}$, where only a few bright member galaxies in a group can be observed, the characteristic group luminosity is no longer the best choice to estimate the halo mass (see also Old et al. 2014, 2015, for the halo mass estimation comparisons on cluster scales). Instead, they proposed a method that is based on the luminosity of the central galaxy, $L_{c}$, and a luminosity 'GAP', $L_{g a p}$, where the central galaxy is defined to be the brightest in a group, and the luminosity gap is defined as $\log L_{\text {gap }}=\log L_{c}-\log L_{s}$, with $L_{s}$ being the luminosity of the satellite galaxy of some rank (e.g. the brightest, or second brightest satellite). The performance of the halo mass estimate is found to be enhanced by using the 'GAP' information. Comparisons between the true halo masses and the masses estimated with the 'GAP' method in mock catalogs show a typical dispersion of $\sim 0.3$ dex.

In this paper, we modify the halo-based group finder developed by Yang et al. (2005a) by using the 'GAP' information. The structure of the paper is as follows. $\S 2$ describes the samples used in this paper, including the 2MRS galaxy sample and a mock galaxy sample used to evaluate the performance of our group finder. In $\S 3$ we describe our modified halo-based group finder. The performance of our group finder, including completeness, contamination, purity is discussed in $\S 4$, together with the reconstruction of the halo mass function. In $\S 5$ the properties of the group catalog constructed from $2 \mathrm{MRS}$ are detailed and compared to the mock group catalog, and to the SDSS DR7 galaxy group catalog constructed by Yang et al. $(2007,2012)$ in the overlapping region. Finally, we summarize our results in $\S 6$. Unless stated otherwise, we adopt a $\Lambda$ CDM cosmology with parameters that are consistent

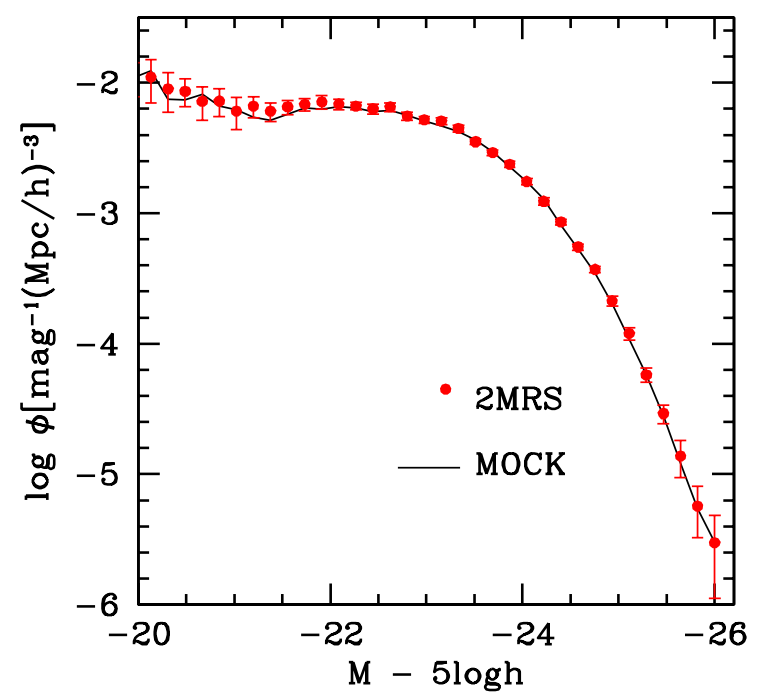

FIG. 1.- Luminosity functions in $K_{s}$ band. Red points are obtained from the 2MRS galaxy sample, while black solid line represents the one obtained from the MOCK sample. The error bars are estimated using 1000 boot-strap re-sampling.

with the nine-year data release of the WMAP mission (hereafter WMAP9 cosmology): $\Omega_{\mathrm{m}}=0.282, \Omega_{\Lambda}=0.718, \Omega_{\mathrm{b}}=$ $0.046, n_{\mathrm{s}}=0.965, h=H_{0} /\left(100 \mathrm{~km} \mathrm{~s}^{-1} \mathrm{Mpc}^{-1}\right)=0.697$ and $\sigma_{8}=0.817$ (Hinshaw et al. 2013).

\section{DATA \\ 2.1. The $2 M R S$ galaxy catalog}

The 2MASS Redshift Survey (2MRS) is based on the Two Micron All Sky Survey (Jarrett et al. 2000, 2003) and is complete to a limiting magnitude of $K_{s}=11.75$, and $\sim 97.6 \%$ of the galaxies brighter than the limiting magnitude have measured redshifts. The survey covers $\sim 91 \%$ of the full sky; only $\sim 9 \%$ of the sky close to the Milky Way plane is excluded (Huchra et al. 2012). The catalog contains about 43,533 galaxies extending out to $\sim 30,000 \mathrm{~km} \mathrm{~s}^{-1}$. For our analysis we only use the 43,246 galaxies with $z \leq 0.08$. Among these, 25 entries have negative redshifts $(-0.001 \leqslant z<0.0)$ which are caused by the peculiar velocities of galaxies. In our analysis, all redshifts are corrected to the Local Group rest frame according to Karachentsev \& Makarov (1996). We also use the distance information provided by Karachentsev et al. (2013) for some nearby galaxies, including 22 galaxies with negative redshifts in our $2 \mathrm{MRS}$ catalog, to reduce effects caused by peculiar velocities. Corrections of Virgo infall are made to 15 galaxies in the front and back of the Virgo cluster according to Karachentsev et al. (2014). Since the redshifts of our $2 \mathrm{MRS}$ galaxies are low, no attempt is made to apply any $K$ - or $E$-corrections to galaxy luminosities.

From this catalog, we first measure the galaxy luminosity function (LF) in the $K_{s}$ band. We adopt the commonly used $1 / V_{\max }$ algorithm (Schmidt 1968; Felten 1976), in which each galaxy is assigned a weight given by the maximum comoving volume within which the galaxy could be observed. Fig 1 shows the galaxy luminosity function so obtained from our 2MRS sample, with the error bars estimated from 1,000 bootstrap re-samplings. We have fitted the LF to a Schechter function (Schechter 1976) and the best fit Schechter parameters are $\log \phi^{*}=1.08 \times 10^{-2}, \alpha=-1.02$ and $M^{*}=$ 
TABLE 1

COMPARISON BETWEEN THREE SAMPLES

\begin{tabular}{lcccc}
\hline \hline Sample & Parent Catalog & Group Finder & Halo Mass & Label \\
\hline MOCKt & mock 2MRS catalog & none & simulation & $M_{\mathrm{t}}$ \\
MOCKg & mock 2MRS catalog & halo-based & Gap model & $M_{\mathrm{g}}$ \\
2MRS & 2MRS catalog & halo-based & Gap model & $M_{2 \mathrm{MRS}}$ \\
\hline
\end{tabular}

-23.55 . These values are consistent with those obtained by Crook et al. (2007) and Tully (2015).

\subsection{The mock $2 M R S$ galaxy catalog}

We construct a mock $2 \mathrm{MRS}$ galaxy catalog to test the performance of our group finder and the reliability of the final galaxy group catalog. The mock catalog is constructed as follows.

First, we use a high-resolution simulation carried out at the High Performance Computing Center, Shanghai Jiao Tong University, using L-GADGET, a memory-optimized version of GADGET-2 (Springel et al. 2005). A total of $3072^{3}$ dark matter particles were followed in a periodic box of $500 h^{-1} \mathrm{Mpc}$ on a side ( $\mathrm{Li}$ et al. 2016). The adopted cosmological parameters are consistent with those from WMAP9. Each particle in the simulation has a mass of $3.4 \times$ $10^{8} h^{-1} \mathrm{M}_{\odot}$. Dark matter halos were identified using the standard FOF algorithm (Davis et al. 1985) with a linking length of $b=0.2$ times the mean inter particle separation.

Next, the halos are populated with galaxies of different luminosities. We use the conditional luminosity function (CLF, Yang, Mo \& van den Bosch 2003), which is defined to be the average number of galaxies, as a function of luminosity, that reside in a halo of a given mass, to link galaxies with dark matter haloes. We make use of the set of CLF parameters provided by Cacciato et al. (2009) to generate model galaxies with $r$ band luminosities. Following the observational definition, the central galaxy is defined as the brightest member and is assumed to be located at the center of the corresponding halo. Its velocity follows the velocity of the dark matter halo center. Other galaxies, referred to as satellite galaxies, are distributed spherically following a NFW (Navarro, Frenk \& White 1997) profile where the concentration model of Zhao et al. (2009) was adopted. Their velocities are assumed to be the sum of the velocity of the host halo center plus a random velocity drawn from a Gaussian distribution with dispersion given by the virial velocity dispersion of the halo. We refer the reader to Lu et al. (2015) and Yang et al. (2004) for details. In general, one can also populate/generate mock galaxies using more sophisticated methods, e.g., based on sub-halos or halo merger trees, where the galaxies are not spherically distributed. However, as we have tested in Weinmann et al. (2006b), our group finder is not very sensitive to the somewhat non-spherical distribution of galaxies.

In order to convert the $r$ band magnitude to the $K_{s}$ band, we first measure the cumulative luminosity function separately for both the mock and 2MRS samples. Assuming that galaxies more luminous in the $r$ band are also more luminous in the $K_{s}$, we assign a $K_{s}$ band luminosity (absolute magnitude) to each galaxy. In practice, we relate $M_{r}$ and $M_{K_{s}}$ through abundance matching:

$$
\int_{-\infty}^{M_{r}} \phi_{r}\left(M_{r}^{\prime}\right) d M_{r}^{\prime}=\int_{-\infty}^{M_{K_{s}}} \phi_{K_{s}}\left(M_{K_{s}}^{\prime}\right) d M_{K_{s}}^{\prime},
$$

where $\phi_{r}\left(M_{r}\right)$ and $\phi_{K_{s}}\left(M_{K_{s}}\right)$ are the luminosity functions of galaxies in $M_{r}$ and $M_{K_{s}}$, respectively.

Finally, we place a virtual observer at the center of our simulation box and define a $(\alpha, \delta)$-coordinate frame, and remove all galaxies that are located outside the survey region $(\sim 9 \%$ of the total sky). We then assign to each galaxy a redshift and an apparent magnitude according to its distance and luminosity, and select only galaxies that are brighter than the magnitude limit $K_{s}=11.75$. Here again, no $\mathrm{K}+\mathrm{E}$ corrections are made to galaxy luminosities. In total, we have 41,876 galaxies in our mock 2MRS catalog. The black solid curve in Fig. 1 shows the $K_{s}$ band luminosity function estimated from our mock sample.

Apart from the luminosity function of galaxies, we set out to measure the true halo mass function in the mock 2MRS catalog. Because of the survey magnitude limit, faint galaxies formed in low mass halos might not be observed at high redshift, i.e., low mass halos can only be detected below a redshift limit. To properly estimate the halo mass function, one needs to have a complete sample of groups (halos), i.e. to obtain the limiting redshift for a given mass of halos, within which the selection of groups is complete. Unlike the luminosity for which the limiting redshift can be directly calculated from the magnitude limit, we use an empirical way to get the limiting redshift for halos. First, we calculate the number densities of halos in small redshift and halo mass bins and plot them in the $\log M_{\mathrm{t}}-z$ plane using color bars (see Fig. 2). We can see that the number density of halos of given mass drops sharply above certain redshift. Here we define the limiting redshift for a given mass halos as the redshift at which this rapid drop in density occurs. The smooth line in Fig. 2 shows the limiting redshift as a function of $\log M_{\mathrm{t}}$ we use, which clearly represents a conservative cut to ensure completeness. Once a limiting redshift is adopted, we can calculate the halo mass function using only halos (and volume) below this redshift. Fig. 3 shows the halo mass function obtained in this way with dots and error bars. Here again, the error bars are estimated using 1000 bootstrap re-samplings. For comparison, we also show, using the solid line, the theoretical model predictions given by Tinker et al. (2008). Compared with the model prediction, the data points are slightly lower at intermediate to low mass range, which is mainly due to cosmic variance, since the overall halo mass function in the whole simulation box is quite consistent with theoretical predictions (see Li et al. 2016).

Since we have both the true halo and the galaxy membership informations in our mock 2MRS sample, we can use it to test the performance of our group finder. Together with the 2MRS observational sample, there are three galaxy group catalogs involved in this paper. We refer to the two catalogs related with the mock 2MRS samples as 'MOCKt' and 'MOCKg' respectively. The former catalog indicates the true group memberships in the FOF dark matter halos obtained directly from the simulation, where we use $M_{\mathrm{t}}$ to represent the true halo mass. The latter is constructed using our group 


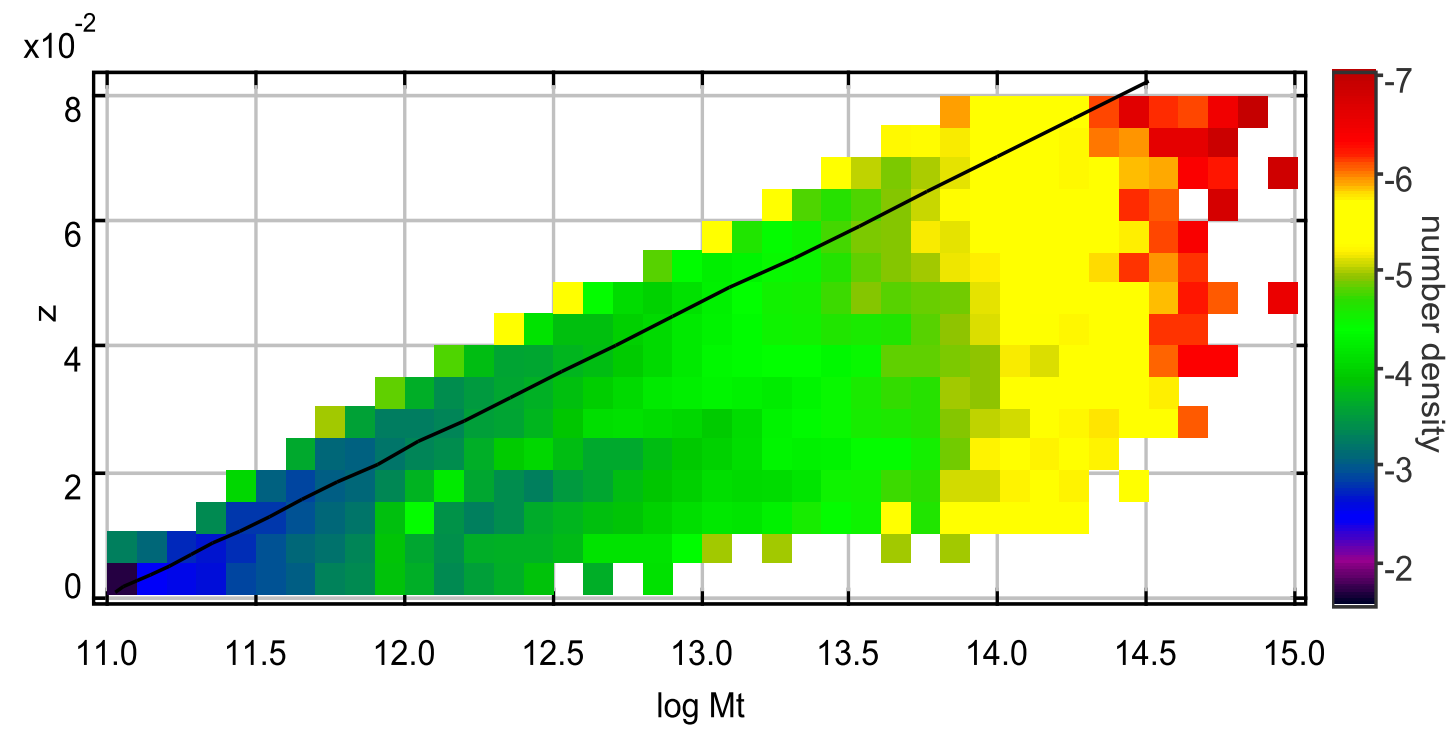

FIG. 2.- The number densities of halos, $\log \phi\left[\left(h^{-1} \mathrm{Mpc}\right)^{-3}\right]$, in each halo mass and redshift bins shown with color bars. The solid line represents a conservative redshift limit $z_{\text {limit }}=0.023 * \log M_{\mathrm{t}}-0.26$, below which a complete sample can be formed for halos with masses down to the mass given by the value of $M_{\mathrm{t}}$ shown by the horizontal axis.

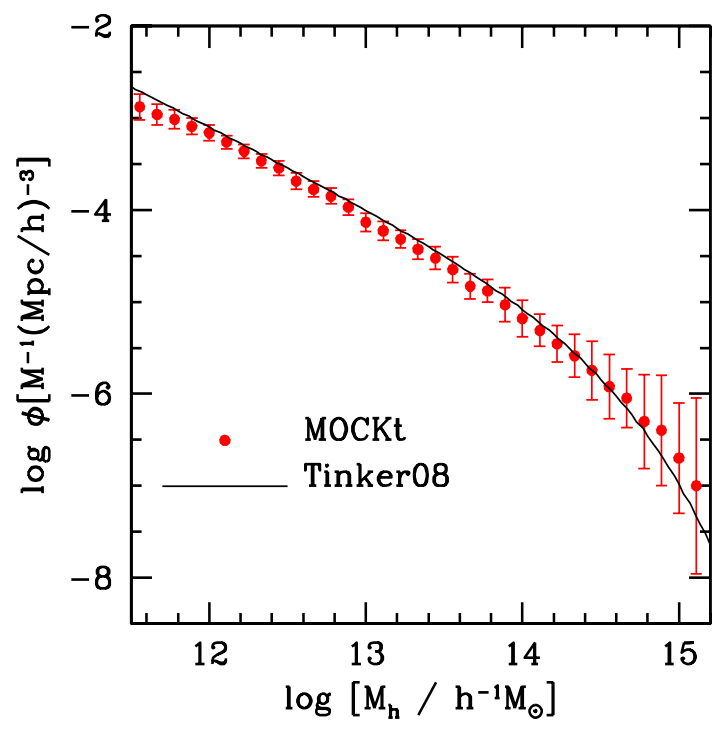

FIG. 3.- The halo mass function of our true sample (MOCKt, dots with error bars). The halo mass function given by Tinker et al. (2008) is also plotted in the same panel for comparison using black solid line.

finder, where the halo masses are estimated using our 'GAP' related mass estimator and are named as $M_{\mathrm{g}}$. Finally, the group catalog constructed from the 2MRS data is referred to as '2MRS' and the related halo mass are named as $M_{2 \mathrm{MRS}}$. For clarity, we list the differences of the three definitions in Table. 1, including the group finders that were used to identify galaxy groups, and the methods used to estimate the halo masses. Apart from the above three specific halo mass definitions, we use $M_{h}$ to represent the general halo masses, including those used in theoretical model predictions (e.g. Tinker et al. 2008).

\section{THE MODIFIED HALO-BASED GROUP FINDER}

One of the key steps in the halo-based group finder (Yang et al. 2005a, 2007) is to have accurate estimates of the halo masses of candidate galaxy groups. As demonstrated in Yang et al. (2007), halo mass is tightly correlated with the total luminosity of member galaxies. In practice, however, one can only estimate a characteristic luminosity which is the sum of the luminosities of member galaxies brighter than some given limit. For a relatively deep survey such as the SDSS, where the limit can be set sufficiently low, the characteristic luminosity is a good proxy of the total luminosity and so can be used to indicate halo mass. For a shallow survey like the 2MRS, on the other hand, only a few (in most cases one or two) brightest member galaxies in the halos can be observed. The characteristic luminosity is no longer the best halo mass estimator, and an alternative is needed. In this paper, we implement the 'GAP' method proposed by Lu et al. (2015).

\subsection{The GAP halo mass estimator}

In the 'GAP' method, one first needs to estimate the $L_{c}-M_{h}$ relation. For MOCKt samples, since every groups have the true central galaxies and true halo masses from the simulation, we can obtain this relation directly. Hereafter we refer the $L_{c}-M_{h}$ relation obtained directly from the simulation as the intrinsic (true) relation. On the other hand, for observational samples, one can obtain this relation from the conditional luminosity function model (e.g. Yang, Mo \& van den Bosch 2003) or from halo abundance matching (e.g. Mo et al. 1999; Vale \& Ostriker 2006; Conroy et al. 2006; Behroozi et al. 2010; Guo et al. 2010). Here we adopt the latter and assume that there is a monotonic relation between the luminosity of central galaxy and the mass of dark matter halo, so that a more luminous galaxy resides a more massive halo. We can then get an initial estimate of the dark matter halo mass for each 
TABLE 2

PARAMETERS OF THE $\Delta \log M_{g}$ MODEL OBTAINED FROM MOCK 2 MRS SAMPLE. [SEE EQS. (4) \& (5)]

\begin{tabular}{lccccc}
\hline \hline$\Delta \log M_{g}$ & $\beta_{1}$ & $\alpha_{2}$ & $\beta_{2}$ & $\beta_{3}$ & $\gamma_{3}$ \\
\hline MEMBER 2 & $10.81_{-0.19}^{+0.18}$ & $0.36_{-0.26}^{+1.60}$ & $-15.44_{-7.86}^{+3.35}$ & $10.39_{-0.24}^{+0.11}$ & $1.94_{-0.41}^{+0.83}$ \\
MEMBER 3 & $10.21_{-0.10}^{+0.39}$ & $0.23_{-0.14}^{+0.54}$ & $-13.40_{-3.97}^{+1.21}$ & $9.90_{-0.10}^{+0.36}$ & $2.21_{-0.27}^{+0.32}$ \\
MEMBER 4 & $9.98_{-0.18}^{+0.32}$ & $0.20_{-0.09}^{+0.25}$ & $-13.39_{-2.76}^{+1.10}$ & $9.81_{-0.17}^{+0.30}$ & $2.45_{-0.15}^{+0.24}$ \\
MEMBER 5 & $9.77_{-0.07}^{+0.33}$ & $0.13_{-0.01}^{+0.15}$ & $-13.67_{-0.94}^{+1.18}$ & $9.67_{-0.07}^{+0.28}$ & $2.54_{-0.08}^{+0.15}$
\end{tabular}

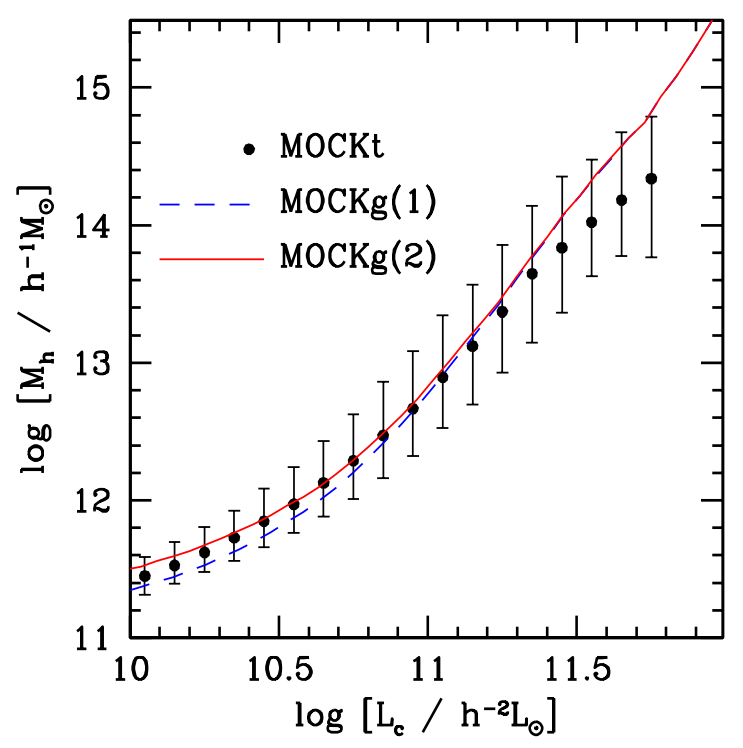

FIG. 4.- $L_{c}-M_{h}\left(M_{\mathrm{g}}\right)$ relation given by the MOCKg sample using abundance matching between the cumulative luminosity function of galaxies and the halo mass function. 'Round 1' relation is obtained using all mock galaxies [blue dashed line, labelled as $\operatorname{MOCKg}(1)]$ while 'Round 2' is obtained using central galaxies only [red line labelled as $\operatorname{MOCKg}(2)]$. The true $L_{c}-M_{h}\left(M_{\mathrm{t}}\right)$ relation given by the MOCKt sample is plotted with black solid points with error bars which indicate the $16 \%-84 \%$ percentiles of the distributions around the median values.

central galaxy from

$$
\int_{L_{c}}^{\infty} n_{c}\left(L_{c}^{\prime}\right) d L_{c}^{\prime}=\int_{M_{h}}^{\infty} n_{h}\left(M_{h}^{\prime}\right) d M_{h}^{\prime},
$$

where, $n_{c}\left(L_{c}\right)$ is the number density of central galaxies with luminosity $L_{c}$ and $n_{h}\left(M_{h}\right)$ is the number density of halos (or halo mass function) with mass $M_{h}$. In this paper, we adopt theoretical halo mass function given by Tinker et al. (2008). Note that, in this abundance matching approach, we also need to know whether a galaxy is a central or a satellite. Since we are trying to find galaxy groups within the observation (the $2 \mathrm{MRS}$ in our case), we can easily separate galaxies into centrals and satellites with the help of group memberships. As we will show later, although the $L_{c}-M_{h}$ relation we obtain may deviates from the true one, especially at the massive end, the deviation can be compensated to some extent by our 'GAP'-based correction factor.

Our modeling of the $L_{c}-M_{h}$ relation using Eq. (2) is carried out via the following two steps. First, before we are able to separate galaxies into centrals and satellites with the help of group memberships, we assume that all of them are centrals (as shown in Yang et al. 2008, more than $60 \%$ of the galaxies are centrals). To show the performance, We have applied this to our mock $2 \mathrm{MRS}$ sample, and obtain the 'Round 1' $L_{c}-M_{\mathrm{g}}$ relation, which is shown in Fig. 4 as the blue dashed line.

For comparison, we also plot, as black solid points, the true median $L_{c}-M_{\mathrm{t}}$ relation obtained from the true centrals and true halo masses in the simulation, with error bars indicate the $16 \%-84 \%$ percentiles of the distributions. Compared to the true relation, we see that, the Round 1 relationship shows a general agreement with the true one, with a slight over-prediction of the halo masses at the bright end and slight under-prediction at the faint end. The deviation at the massive end is caused by the Malmquist bias in the $L_{c}-M_{\mathrm{g}}$ relation which can be corrected by the 'GAP' (see Lu et al. 2015). The deviation at the faint end is caused by the inclusion of all the galaxies (including satellites) in our abundance matching. As we apply our group finder to the galaxy catalog in the next step, the group membership will enable us to separate galaxies into centrals and satellites. We can then limit the application of the abundance matching to centrals only, and improve the $L_{c}-M_{h}$ relation. After two to three iterations we converge to a new set of group memberships and a new $L_{c}-M_{\mathrm{g}}$ relationship, which is referred to as 'Round 2' and shown as the solid red line in Fig. 4. After this step, there is no longer any systematic deviation of the $L_{c}-M_{\mathrm{g}}$ relationship relative to the true one at the low mass end.

With the $L_{c}-M_{\mathrm{g}}$ obtained in this step, we can estimate the 'luminosity gap', which is defined as the luminosity ratio between the central and a satellite galaxy in the same halo, $\log L_{\text {gap }}=\log \left(L_{c} / L_{s}\right)$ (see Lu et al. 2015). The halo mass is then estimated using the relation,

$$
\log M_{\mathrm{g}}\left(L_{c}, L_{\text {gap }}\right)=\log M_{\mathrm{g}}\left(L_{c}\right)+\Delta \log M_{\mathrm{g}}\left(L_{c}, L_{\text {gap }}\right) .
$$

This halo mass estimator consists of two parts. The first part is an empirical relation between $M_{\mathrm{g}}$ and $L_{c}$ derived from Eq. (2) which is represented by the first term on the right side. Another part is the amount of correction to that relation, which is represented by the second term $\Delta \log M_{\mathrm{g}}\left(L_{c}, L_{\text {gap }}\right)$. In order to model this correction term, we use the following functional form,

$$
\Delta \log M_{\mathrm{g}}\left(L_{c}, L_{\text {gap }}\right)=\eta_{a} \exp \left(\eta_{b} \log L_{\text {gap }}\right)+\eta_{c} .
$$

The parameters $\eta_{a}, \eta_{b}$ and $\eta_{c}$ all depend on $L_{c}$ as:

$$
\eta_{a}\left(L_{c}\right)=\exp \left(\log L_{c}-\beta_{1}\right)
$$




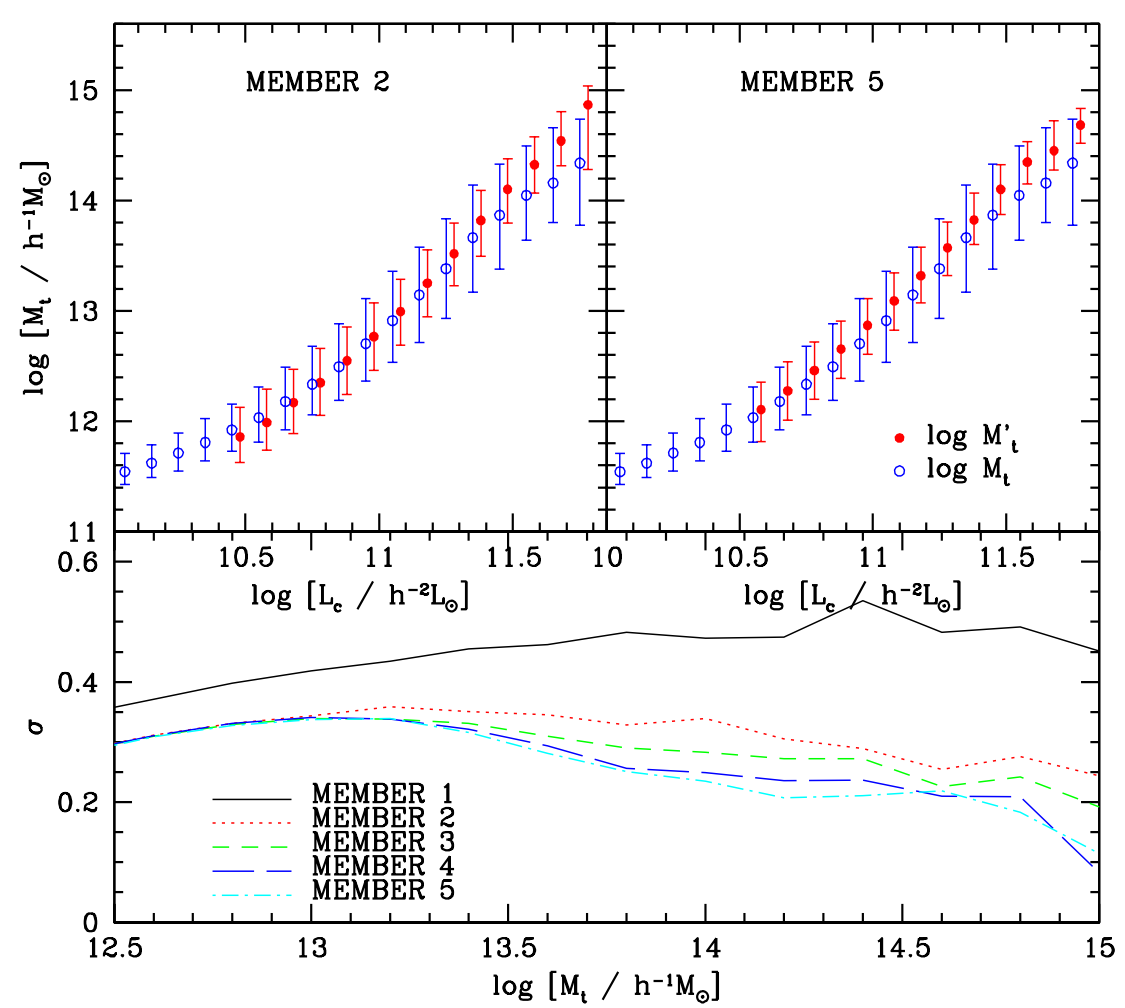

FIG. 5.- Comparison between the original (open blue circles) $\log M_{\mathrm{t}}$ and corrected (solid circles) $\log M_{\mathrm{t}}^{\prime}$ halo masses by using the luminosity gap between the central (brightest) and the second brightest (top left) and the fifth brightest member galaxies (top right), respectively. The error bars indicate the $16 \%-84 \%$ percentiles of the distributions. The standard variances $\sigma$ between estimated halo mass and the true halo mass are illustrated in the bottom panel. As the legend indicates, results are shown for groups with 2, 3, 4 and 5 members, while the halo masses estimated only by using central galaxies (member 1) are also given in the same panel.

$$
\begin{aligned}
& \eta_{b}\left(L_{c}\right)=\alpha_{2}\left(\log L_{c}+\beta_{2}\right) \\
& \eta_{c}\left(L_{c}\right)=-\left(\log L_{c}-\beta_{3}\right)^{\gamma_{3}}
\end{aligned}
$$

which is specified by five free parameters.

For a given $L_{c}-M_{\mathrm{g}}$ relation, we fit the model to the true halo masses $M_{\mathrm{t}}$ of our galaxy systems (groups) in our mock sample to have the minimum variances between $\log M_{\mathrm{t}}$ and $\log M_{\mathrm{g}}\left(L_{c}, L_{\text {gap }}\right)$ (see Lu et al. 2015, for details). Table 2 presents the set of best fit values of these parameters. Since the (mock) 2MRS sample is shallow, we provide the parameters up to 5 group members. As an illustration, Fig. 5 shows the performance of this halo mass estimator. In the top two panels, the original $L_{c}-M_{t}$ relations are shown as the open circles; the GAP-corrected relations are shown as the solid points, with the left panel showing results for $L_{s}=L_{2}$ and the right for $L_{s}=L_{5}$. To see the improvement, we define a 'pre-corrected' halo mass,

$$
\log M_{\mathrm{t}}^{\prime}=\log M_{\mathrm{t}}-\Delta \log M_{g}\left(L_{c}, L_{\text {gap }}\right) .
$$

If the correction term $\Delta \log M_{\mathrm{g}}\left(L_{c}, L_{\text {gap }}\right)$ can perfectly describe the scatter in the original relation $L_{c}-M_{\mathrm{t}}$, then there would be no scatter in the $L_{c}-M_{\mathrm{t}}^{\prime}$. We can see that, the scatter in the $L_{c}-M_{\mathrm{t}}^{\prime}$ is significantly reduced compare to that in the $L_{c}-M_{\mathrm{t}}$ relation. For massive halos/groups, this improvement is quite notable where the scatter is reduced almost by a factor of two. The bottom panel of Fig. 5 shows the standard deviation $\sigma$ of the halo mass $\log M_{\mathrm{g}}\left(L_{c}, L_{\text {gap }}\right)$ obtained by Eq.(3) from the true halo mass $\log M_{\mathrm{t}}$. In both Lu et al. (2015) and this paper, we find that using $L_{5}$ gives the best correction to the halo mass. As shown Lu et al. (2015), such a correction factor is quite independent of the galaxy formation model used to construct the mock catalog. In this paper we use the set of best fit parameters only up to the fifth ranked member (see below).

\subsection{The Group Finder}

The group finder adopted here is similar to that developed by Yang et al. (2005a). It uses the general properties of dark matter haloes, namely size and velocity dispersion, to iteratively find galaxy groups. Tests show that this group finder is powerful in linking galaxies with dark matter halos, even in the case of single member groups. As we pointed out earlier, the halo mass estimation adopted in Yang et al. (2005a, 2007 ) is based on the ranking of the characteristic group luminosity and proves to be quite reliable for surveys like the 2dFGRS and SDSS. For the 2MRS considered here, we use the 'GAP'-corrected estimator described above. The modified group finder with this halo mass estimator consists of the following main steps:

\section{Step 1: Start the halo-based group finder.}

In the earlier version of the halo-based group finder, the first step is to use the FOF algorithm (Davis et al. 1985) with very small linking lengths in redshift space to find potential groups. Here we assume all galaxies in our catalog are candidate groups. The halo mass of each candidate group is calculated using the $L_{c}-M_{h}$ relation obtained in Eq. (2) (Round 
1).

Step 2: Update group memberships using halo information.

After assigning halo masses to all the candidates, groups are sorted according to their halo masses. Starting from the most massive one, we estimate the size and velocity dispersion of the dark matter halo, using the halo mass currently assigned to it. A dark matter halo is defined to have an over-density of 180. For the WMAP9 cosmology adopted here, the radius is approximately

$$
r_{180}=1.33 h^{-1} \mathrm{Mpc}\left(\frac{M_{h}}{10^{14} h^{-1} \mathrm{M}_{\odot}}\right)^{1 / 3}\left(1+z_{\text {group }}\right)^{-1},
$$

Here, $z_{\text {group }}$ is the redshift of the group center. The line-ofsight velocity dispersion of the halo is

$$
\sigma=418 \mathrm{~km} \mathrm{~s}^{-1}\left(\frac{M_{h}}{10^{14} h^{-1} \mathrm{M}_{\odot}}\right)^{0.3367} .
$$

Finally, following Yang et al. (2007, hereafter Y07), we use the luminosity weighted center of member galaxies as the new group center.

Then, one can assign new member galaxies to the group according to the tentative group center, tentative estimates of halo size and velocity dispersion obtained in the above steps. The phase-space distribution of galaxies is assumed to follow that of dark matter, and the group center is assumed to coincide with the center of halo. We use the following function of the projected distance $R$ and $\Delta z=z-z_{\text {group }}$ to describe the number density of galaxies at $z$ in the redshift space around the group center at redshift $z_{\text {group }}$ :

$$
P_{M}(R, \Delta z)=\frac{H_{0}}{c} \frac{\Sigma(R)}{\bar{\rho}} p(\Delta z),
$$

where $c$ is the speed of light and $\bar{\rho}$ is the average density of the Universe. We assume the projected surface density, $\Sigma(R)$, is given by a (spherical) NFW (Navarro, Frenk \& White 1997) profile:

$$
\Sigma(R)=2 r_{s} \bar{\delta} \bar{\rho} f\left(R / r_{s}\right),
$$

where $r_{s}$ is the scale radius, and the shape function is

$$
f(x)=\left\{\begin{array}{ll}
\frac{1}{x^{2}-1}\left(1-\frac{\left.\ln \frac{1+\sqrt{1-x^{2}}}{\sqrt{1-x^{2}}}\right)}{}\right) & \text { if } x<1 \\
\frac{1}{3} & \text { if } x=1 \\
\frac{1}{x^{2}-1}\left(1-\frac{\operatorname{atan} \sqrt{x^{2}-1}}{\sqrt{x^{2}-1}}\right) & \text { if } x>1
\end{array} .\right.
$$

The normalization of the profile depends on the concentration $c_{180}=r_{180} / r_{s}$ as:

$$
\bar{\delta}=\frac{180}{3} \frac{c_{180}^{3}}{\ln \left(1+c_{180}\right)-c_{180} /\left(1+c_{180}\right)},
$$

where the concentration model of Zhao et al. (2009) is adopted. The redshift distribution of galaxies within the halo is assumed to have a normal distribution, and can be described as follows,

$$
p(\Delta z)=\frac{1}{\sqrt{2 \pi}} \frac{c}{\sigma\left(1+z_{\text {group }}\right)} \exp \left[\frac{-(c \Delta z)^{2}}{2 \sigma^{2}\left(1+z_{\text {group }}\right)^{2}}\right],
$$

where $\sigma$ is the rest-frame velocity dispersion given by equation (8). So defined, the three-dimensional density in redshift space is $P_{M}(R, \Delta z)$. Then, we apply the following procedures to assign a galaxy to a particular group. For each galaxy we loop over all groups, and compute the distances $R$ and $\Delta z$ between the galaxy and the group center. An appropriately chosen background level $B=10$ is applied to the density contrast for galaxies to be assigned to a group. If, according to this criterion, a galaxy can be assigned to more than one group it is only assigned to the one with the highest $P_{M}(R, \Delta z)$. Finally, if all members of two groups can be assigned to one, they are merged into a single group. Note that in our group finder, the background parameter $B=10$ is set to ensure the balance between the interlopers and completeness of group memberships. A lower $B$ value will increase both the completeness of the group memberships and the number of interlopers, especially in massive groups. Thus for those who care most about the completeness of the group memberships only, a lower value of $B$ (e.g. $B=5$ ) can be used (see Yang et al. 2005a).

\section{Step 3: Update halo mass with 'GAP' correction.}

Once the new membership to a group is obtained, we use the new central and satellite galaxy system to estimate the halo mass using the 'GAP' method described by Eq. (3). For each candidate group, we use the $L_{c}-M_{h}$ relation and the luminosity gap $\log L_{\text {gap }}$ between the central galaxy and the faintest satellite (if the group contains less than 5 members) or the fifth brightest galaxy (if the group has membership equal to or larger than 5), to estimate the halo mass. In practice, we only apply the luminosity gap correction for centrals in the luminosity range $10.5 \leq \log L_{c} \leq 11.7$. As shown in the top panels of Fig. 5, fainter $\left(\log \bar{L}_{c} \leq 10.5\right)$ central galaxies are basically isolated. For $\log L_{c} \geq 11.7$, we found that using the value of $L_{c}$ directly in the GAP leads to overcorrelation. Thus, for these systems we set $\log L_{c}=11.7$ to estimate the GAP correction. In addition, since our galaxy sample is magnitude limited to $K_{s}=11.75$, our method also suffers from a 'missing satellite' problem, in that some groups do not contain any satellites brighter than the magnitude limit. As an attempt to partly correct for this, we assume that each galaxy group that contains only one member (a central) has a potential member satellite galaxy with an apparent magnitude $K_{s}=11.75$, which corresponds to a limiting luminosity $L_{\text {limit }}$ at the distance of the group. A 'GAP' correction, $\log L_{c}-\log L_{\text {limit }}$, is also applied to all groups of single membership with $\log L_{c}-\log L_{\text {limit }} \geq 0.5$, and the final halo mass of such a group is set to be the average value between this mass and the original mass based on the central galaxy alone.

\section{Step 4: Update the $L_{c}-M_{h}$ relation and Iterate.}

Once all the groups have been updated for new memberships, we can distinguish between centrals and satellites. We use the updated central galaxy sample to update the $L_{c}-M_{h}$ relation (Round 2) to be used to assign halo masses to tentative groups. We iterate Steps 2-4 until convergence is reached. Typically three iterations are needed to achieve convergence. Our final catalog is the collection of all the converged groups with information about their positions, galaxy memberships, and halo masses. 


\section{Step 5: Update the final halo masses of groups.}

Once all the groups (memberships) have been finalized, we perform a final update of the halo masses of groups using an abundance matching method so that the halo mass function of the groups is consistent with theoretical predictions (e.g. Yang et al. 2007). In performing the halo abundance matching, we measure the cumulative halo mass functions of groups following the procedures described in section 2.2.

\section{TEST WITH MOCK CATALOGS}

In this section, we test the performances of our group finder, both in halo masses and group memberships it assigns, by comparing the groups selected by our group finder (MOCKg) with the true groups defined by simulation (MOCKt) in our mock 2 MRS sample.

\subsection{Completeness, Contamination and Purity}

Starting from a total of 41,876 galaxies in the mock $2 \mathrm{MRS}$ sample, our group finder returns 32,368 galaxy groups, among which 4,225 have 2 or more members, and the rest only one member. We follow Yang et al. (2005a, 2007) to assess the performance of the group finder. The procedure is as follows. First, for each group, $k$, in the MOCKg sample, we identify the halo with ID, $h_{k}$, in MOCKt according to its brightest member. Then, we define the total number of true members belong to halo $h_{k}$ to be $N_{t}$. Among $N_{t}$, the number of true members that belong to the group $k$ is written as $N_{s}$. The number of interlopers (group members that belong to a different halo) in the group $k$ is defined as $N_{i}$, while the total number of group members selected by our group finder in $\mathrm{MOCKg}$ is assumed to be $N_{g}$, and $N_{g}=N_{i}+N_{s}$. If our group finder is perfect, it should have $N_{i}=0$ and $N_{t}=N_{s}=N_{g}$. With these numbers, we can define the following three quantities:

- COMPLETENESS: $f_{c} \equiv N_{s} / N_{t}$;

- CONTAMINATION: $f_{i} \equiv N_{i} / N_{t}$;

- PURITY: $f_{p} \equiv N_{t} / N_{g}$.

Here, $f_{p}=1 /\left(f_{c}+f_{i}\right)$. If the group is incomplete, then the COMPLETENESS $f_{c}<1$. For the CONTAMINATION $f_{i}$, it can be larger than unity. Finally, for PURITY $f_{p}$, when the number of interlopers is larger than the number of missed true members, $f_{p}<1$, on the other hand, if the number of missed true members is lager than the interlopers, then $f_{p}>1$. If our group finder is perfect, then it should have $f_{c}=f_{p}=1$ and $f_{i}=0$ for all the groups. Note also that the value for the background level $B=10$ was tuned to maximize the average value of $f_{\mathrm{c}}\left(1-f_{\mathrm{i}}\right)$, as described in Yang et al. (2005a).

Fig. 6 shows the reliability of our group catalog constructed from the mock $2 \mathrm{MRS}$ galaxy sample. Following Y07, here, only groups in MOCKg with richness $N_{g} \geq 2$ are included since the single groups with only one member always have zero contamination $f_{i}=0$ as defined above. The upper panel shows the cumulative distributions of the COMPLETENESS $f_{c}$. The groups with different true halo mass are represented with different lines as indicated. The fractions of groups with 100 percent completeness $\left(f_{c}=1\right)$ range from $\sim 85 \%$ to $\sim 65 \%$ depends on halo mass, which shows that more massive groups tend to have lower completeness fraction. Since the massive groups with larger velocity dispersions tend to have larger $f_{i}$ due to contamination of foreground and background galaxies, meanwhile, the purpose of our group finder
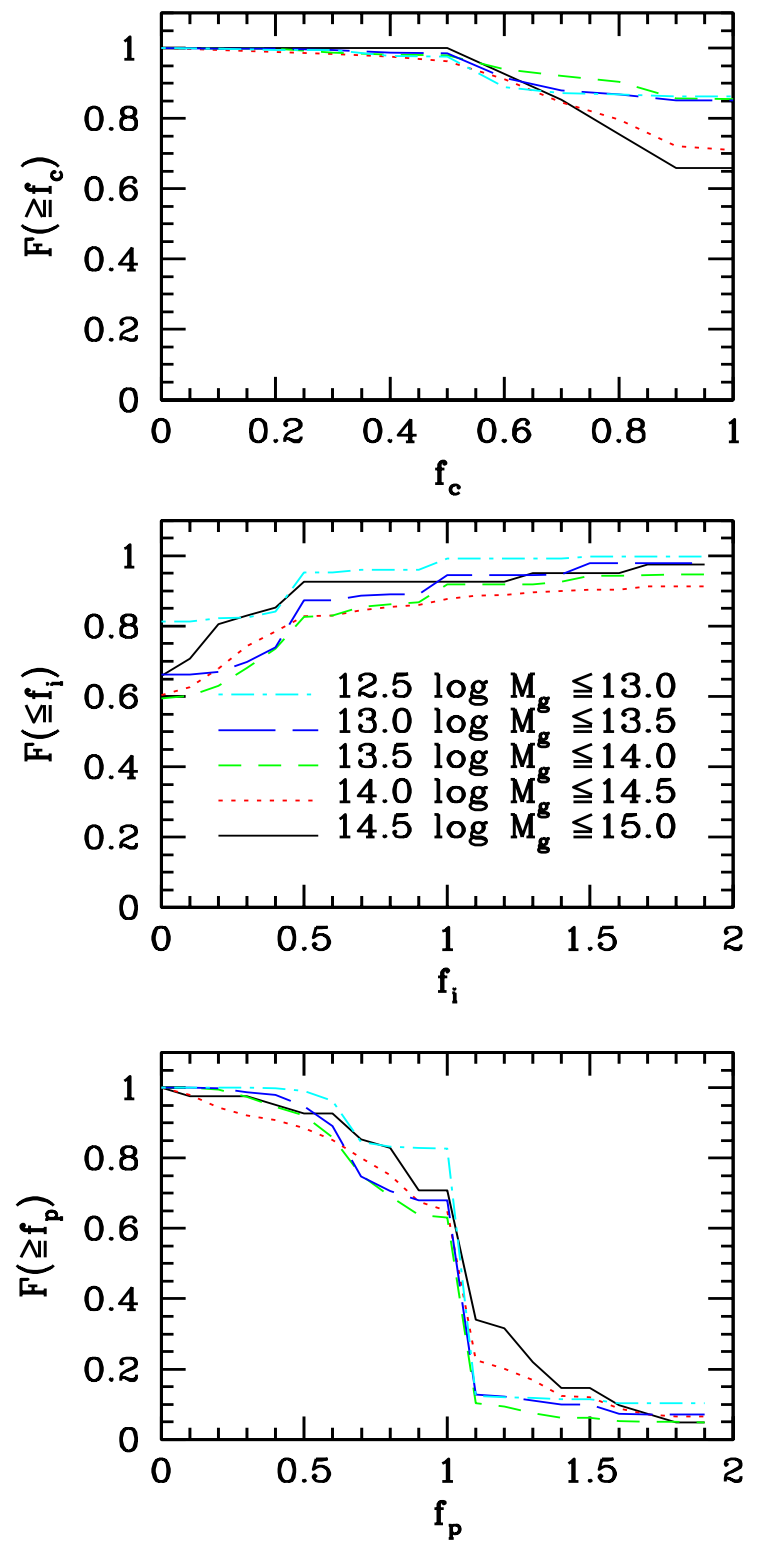

FIG. 6.- The top, middle and bottom panels show the cumulative distributions of completeness, $f_{c}$ (the fraction of true members), contamination, $f_{i}$ (the fraction of interlopers), and purity, $f_{p}$ (ratio between the number of true members and the total number of group members). These values are number weighted. Different lines represent the results for groups in halos of different masses, as indicated. Results are plotted for groups with at least 2 members, since groups with only 1 member have, by definition, $f_{i}=0$.

is to maximizing the average value of $f_{c}\left(1-f_{i}\right)$, a compromise between $f_{c}$ and $f_{i}$, a background level $B=10$ is thus chosen. A smaller $B$ value will increase both $f_{c}$ and $f_{i}$ values in more massive groups, which is not preferably adopted in our investigation. Overall, more than $90 \%$ of our groups have COMPLETENESS $f_{c}>0.6$. For groups with $\log M_{\mathrm{g}} \leq 14.0$, about $80 \%$ of all groups have $f_{c}>0.8$; only for massive halos with $\log M_{\mathrm{g}}>14.0$ is this fraction a little lower, $\sim 75-80 \%$.

The middle panel of Fig. 6 shows the cumulative distribution of the CONTAMINATION $f_{i}$. The fraction of groups with $f_{i}=0$ ranges from $60 \%$ to $80 \%$, depending on the halo 

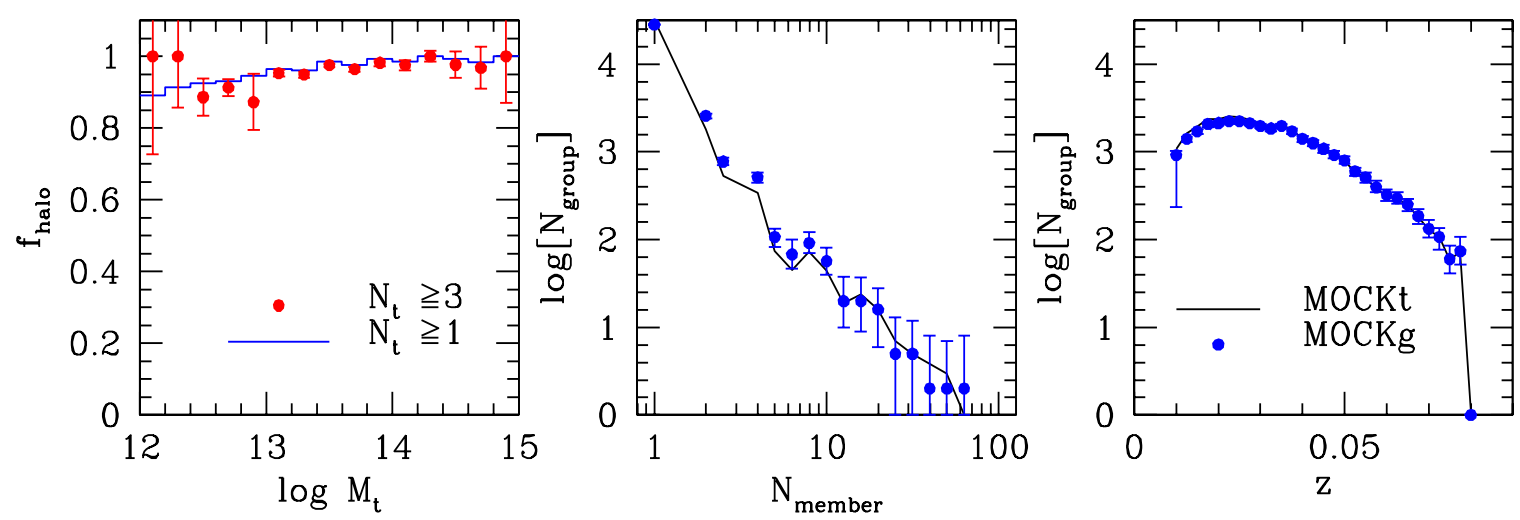

FIG. 7.- Left panel: global completeness $f_{\text {halo }}$, defined as the fraction of halos in the mock sample whose brightest member has actually been identified as the brightest (central) galaxy of its group, as function of the true halo mass $M_{\mathrm{t}}$. Results are shown for all halos (solid blue line) and for those with at least three members in the mock sample (dashed red line). Middle and right panels: the distributions of richness (middle) and redshift (right) of groups. The results obtained from the group catalog constructed from the mock galaxy sample (MOCKg) are represented by solid points. The distributions obtained from true sample (MOCKt) are shown by the solid lines. All the error bars shown in the figure are estimated from 1000 bootstrap re-samplings.

mass, while $\sim 85 \%$ of all the groups have $f_{i}<0.5$. The interlopers producing the contamination are either nearby field galaxies or the member galaxies of nearby massive groups, especially for systems that are along the same line of sight. Although the results for different halo masses are similar, groups in the lowest mass bin seems to have the highest fraction of interlopers.

Finally, the cumulative distribution of the PURITY $f_{p}$ is shown in the lower panel. On average, the number of groups which have $f_{p}<1$ is about the same with that have $f_{p}>1$. The break at $f_{p}=1$ indicates that the number of recovered group members is about the same as the number of the true members. Thus, the sharper the break is, the better. The ideal case, if our group finder is perfect, it should be a step function at $f_{p}=1$. As one can see, only for massive haloes there is a small fraction, $\sim 10 \%$, with $f_{p}<0.5$, and a significant fraction, $\sim 15 \%$, with $f_{p}>1.5$.

We also calculate the COMPLETENESS, CONTAMINATION and PURITY in terms of the total luminosity rather than the number of member galaxies as shown in Fig. 6. Although not explicitly shown here, the corresponding results are very similar to those in terms of the number of member galaxies.

We now turn to the global properties of groups. First, we examine the global completeness, $f_{\text {halo }}$ which defined to be the fraction of halos in the MOCKt whose brightest members have actually been identified as the brightest (central) galaxies of the corresponding groups in the MOCKg. The left panel of Fig. 7 shows $f_{\text {halo }}$ as a function of the true halo mass, obtained from our MOCKt sample for halos with $N_{t} \geq 1$ (solid blue line) and $N_{t} \geq 3$ (dashed red line), respectively. As one can see, more than $90 \%$ of all the true halos with masses $\geq 10^{13} h^{-1} M_{\odot}$ are selected by our group finder, almost independent of their richness. There is a weak trend with halo mass, in the sense that the performance of the group finder, in terms of $f_{\text {halo }}$, is better for more massive halos. The other global properties we examined are the richness and redshift distributions of galaxy groups. Shown in the middle and right panel of Fig. 7 are the two resulting distributions for the MOCKg and MOCKt catalogs, respectively, and good agreement is clearly seen between MOCKg and MOCKt.

\subsection{Halo masses of galaxy groups}

An important aspect of our group finder is the assignment of halo masses to the groups. An accurate halo mass estimate is not only important in determining group memberships according to common dark halos, but also in the applications of our group catalog to the investigations of galaxy populations in halos and large-scale structure traced by galaxy groups. As described above, our halo mass estimate is based on the ranking of the 'GAP' corrected luminosities of central galaxies, and our test in $\S 3.1$ using true halo masses and group membership information in MOCKt shows that this halo mass estimate is unbiased and has scatter typically of 0.35 to 0.2 dex for halos with masses between $\sim 10^{13}$ to $\sim 10^{15} h^{-1} \mathrm{M}_{\odot}$. However, in real applications, the halo mass estimate also suffers from survey selection effects, contamination and incompleteness of group memberships, and so on. The accuracy of the mass estimate is expected to be reduced. Here we check the accuracy of our halo mass estimates in MOCKg catalog which is constructed from the mock $2 \mathrm{MRS}$ sample using our group finder.

Fig. 8 shows the comparison between the true halo mass $M_{\mathrm{t}}$ and the estimated halo mass $M_{\mathrm{g}}$ from the galaxy group catalog we constructed from the mock $2 \mathrm{MRS}$ sample. An estimated group in MOCKg is paired with a true one in MOCKt if they both contains the same central galaxy, and we compare the halo mass assigned by our group finder in $\mathrm{MOCKg}$ with the true halo mass in MOCKt. Note that because of the contamination of our group finder (merger or fragmentation), only about $\gtrsim 90 \%$ groups are paired and shown here (cf. the left panel of Fig. 7). The left panel of Fig. 8 shows the comparison for all groups while the right panels for $\mathrm{MOCKg}$ groups with more than one member $N_{g} \geq 1$. The corresponding standard deviations are plotted in the bottom two panels, with different lines representing groups of different richness. Fig. 8 shows that the deviation is typically between $0.2-0.45$ dex for all groups, with some dependence on halo mass. For $N_{g} \geq 1$, the scatter appears to be the largest for halos with $M_{\mathrm{t}} \sim 10^{13} h^{-1} \mathrm{M}_{\odot}$. The bottom right panel shows that the mass estimate is improved as the group richness increases. For groups with $N \geq 3$, the scatter is about 0.35 dex, which is comparable to that obtained by Y07 for SDSS groups.

The number distribution of groups as a function of halo mass recovered is another important test of the group finder. 


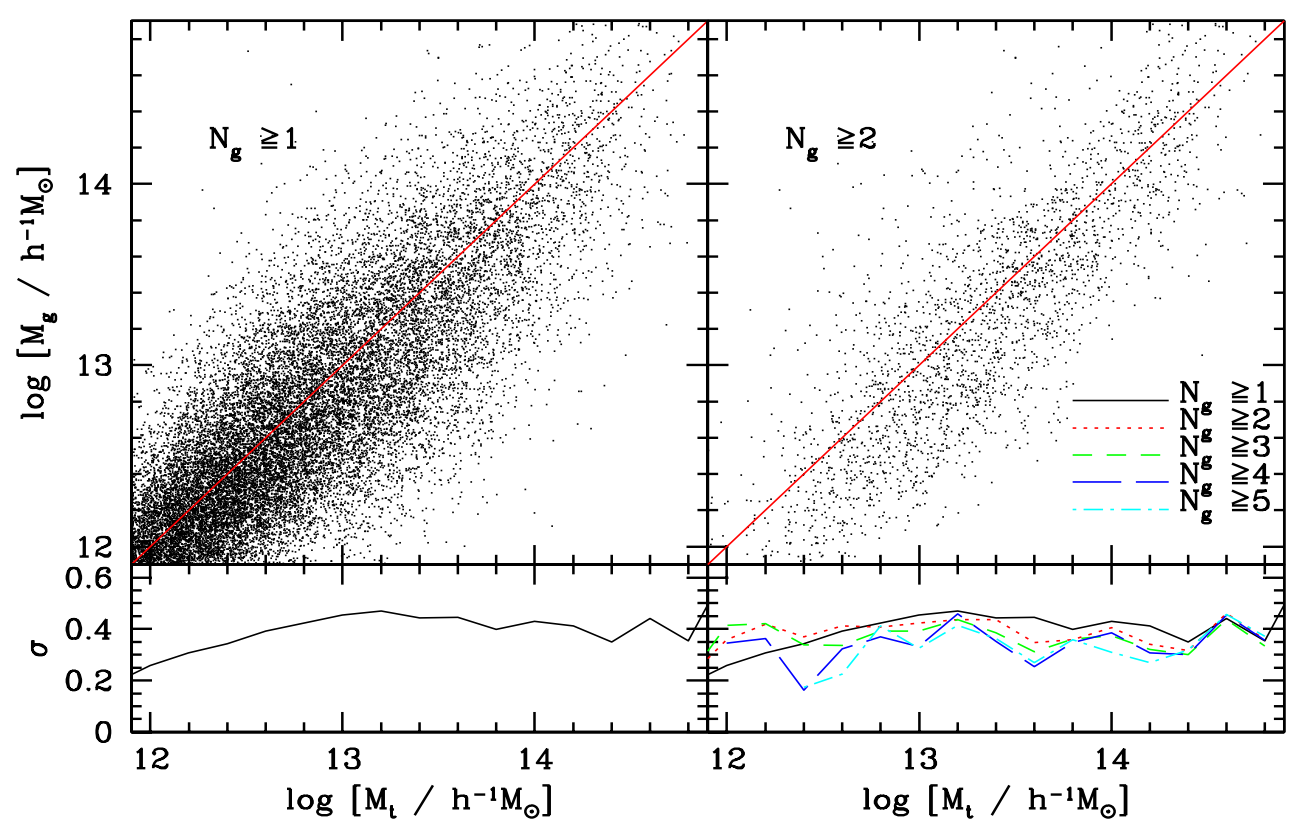

FIG. 8.- Comparison between the estimated halo mass $M_{\mathrm{g}}$ by group finder and the true halo mass $M_{\mathrm{t}}$ in the simulation. Points in top panels are shown for all groups (left) and groups with more than one members (right). Red lines show the relation $M_{\mathrm{g}}=M_{\mathrm{t}}$. The standard variations from the red lines are shown as $\sigma$ in the bottom small panels, with different lines represent results for groups with different richness, as indicated.

In Fig. 9 we show, as the solid points with error bars (obtained by 1000 bootstrap re-samplings), the number distribution of groups as a function of halo mass obtained from the MOCKg catalog selected by our group finder. For comparison, the distribution of true halos given by the MOCKt catalog is shown as the solid line. We see that the number distribution obtained with our group finder matches fairly well with the true halos. The slight over prediction of the number of groups at the intermediate mass range in the MOCKg is caused by the fact that we have forced the final halo mass function of groups to agree with theoretical model prediction, while the real mass function of MOCKt may deviate from the theoretical prediction due to cosmic variance (cf. Fig. 3).

\section{THE 2MRS GALAXY GROUP CATALOG}

We apply our modified group finder to the $2 \mathrm{MRS}$ galaxy catalog in exactly the same way with MOCKg catalog as described in the last section. In the following, we describe our catalog and present some of its basic properties. We also make comparisons with the SDSS groups in the overlapping region, and discuss how some known nearby structures are represented in our catalog.

\subsection{Basic Properties}

Our modified halo-based group finder identifies 29,904 groups from a total of 43,246 2MRS galaxies in the redshift range $z \leq 0.08$. Among the groups selected, 5,286 have two or more members; 2,208 are triplets; and 1,189 have four or more members. Fig. 10 shows the distribution of all groups in the $2 \mathrm{MRS}$ catalog. In the upper panel, the red points represent groups in the redshift range $0.0<z \leq 0.02$, while green and blue points represent groups in $0.02<z \leq 0.03$ and $0.03<$ $z \leq 0.08$, respectively. In the lower panel, red, green and blue points represent groups in mass ranges $\log M_{2 \mathrm{MRS}} \geq 13.5$, $13.5 \geq \log M_{2 \mathrm{MRS}} \geq 12.5$ and $12.5 \geq \log M_{2 \mathrm{MRS}}$, respec-

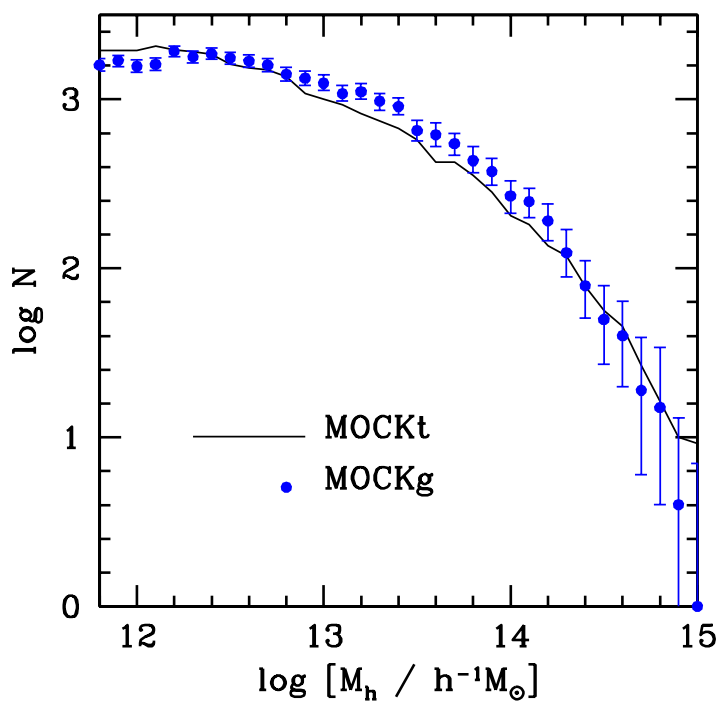

FIG. 9.- The number distribution of groups/halos as a function of halo mass. The results obtained from the group catalog constructed from the mock galaxy sample (MOCKg) are represented by solid points. The error bars are estimated from 1000 bootstrap re-samplings. The black solid curve represents the results obtained using the true halos in the mock 2MRS sample (MOCKt).

tively. One can see from the lower panel that more massive groups seem to locate preferentially denser regions.

Table 3 lists the number of groups in the 2MRS within two redshift ranges: $z=0.01-0.03$ and $z \leq 0.08$, with single member or with more than one member. We also list the number of massive groups with estimated halo masses in two mass ranges, $14.0 \geq \log M_{2 \mathrm{MRS}} \geq 13.0$ and $\log M_{2 \mathrm{MRS}} \geq$ 14.0. For comparison, the number of the mock groups constructed by our group finder (MOCKg) and given by simula- 

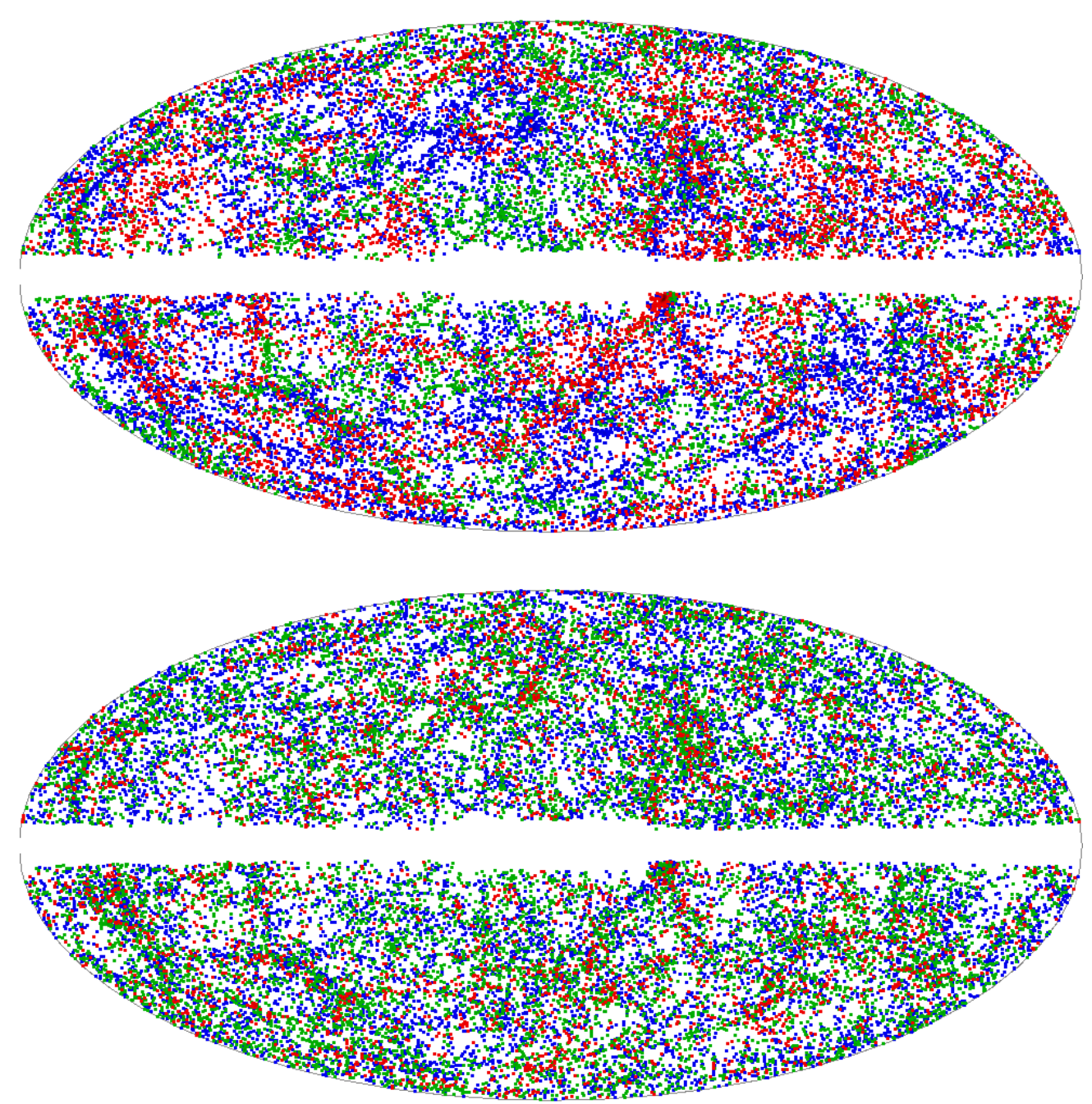

FIG. 10.- The distribution of 2MRS groups in Galactic coordinates, with Galactic longitude increasing from $0^{\circ}$ at the center to $180^{\circ}$ to the left, and from $180^{\circ}$ from the right to $360^{\circ}$ at the center. The Galactic latitude from $-90^{\circ}$ to $90^{\circ}$ from bottom to top. Upper panel: red, green and blue points represent groups within the redshift range: $0.0 \leq z \leq 0.02,0.02 \leq z \leq 0.03$ and $0.03 \leq z \leq 0.08$, respectively. Lower panel: red, green and blue points represent groups within the mass range: $\log M_{2 \mathrm{MRS}} \geq 13.5,13.5 \geq \log M_{2 \mathrm{MRS}} \geq 12.5$ and $12.5 \geq \log M_{2 \mathrm{MRS}}$, respectively.

TABLE 3

PROPERTIES OF 2MRS CATALOGS

\begin{tabular}{lcccccc}
\hline \hline Sample & Galaxies & Groups & $N=1$ & $N \geq 2$ & $14.0 \geq \log M_{h} \geq 13.0$ & $\log M_{h} \geq 14.0$ \\
\hline 2MRS 0.01 $\leq z \leq 0.03$ & 20921 & 12879 & 10004 & 2875 & 1495 & 61 \\
2MRS 0.0 $\leq \leq 0.08$ & 43246 & 29904 & 24618 & 5286 & 8484 & 1103 \\
MOCKg & 41876 & 32368 & 28143 & 4225 & 8085 & 1098 \\
MOCKt & 41876 & 34846 & 31879 & 2967 & 6103 & 867 \\
\hline
\end{tabular}

tion (MOCKt) in the redshift range $z \leq 0.08$ are also listed in Table 3. We show in the left panel of Fig. 11 the richness distribution of groups in $2 \mathrm{MRS}$ which are shown as the dots with error bards. Compare to the MOCKg which is shown as the solid line, the $2 \mathrm{MRS}$ sample tends to contain more rich groups with $N_{g}>32$. However, the total number of such rich systems is small and the statistic is rather poor. The middle panel of Fig. 11 shows the redshift distribution of groups, where the redshift of each group is the luminosity-weighted average of the redshifts of its member galaxies. Here we see that the 2MRS sample contains slightly less groups at low redshift and slightly more groups at high redshift than the mock 2MRS sample.

One of the purposes of constructing the 2MRS galaxy group catalog is to populate the local Universe with well estimated dark matter halos for our subsequent reconstructions of the local density field (e.g. Wang et al. 2014). We check the number distribution of galaxy groups as a function of halo mass in the $2 \mathrm{MRS}$ volume which are shown in the right panel of Fig. 11 using black points with error bars. For comparison, 


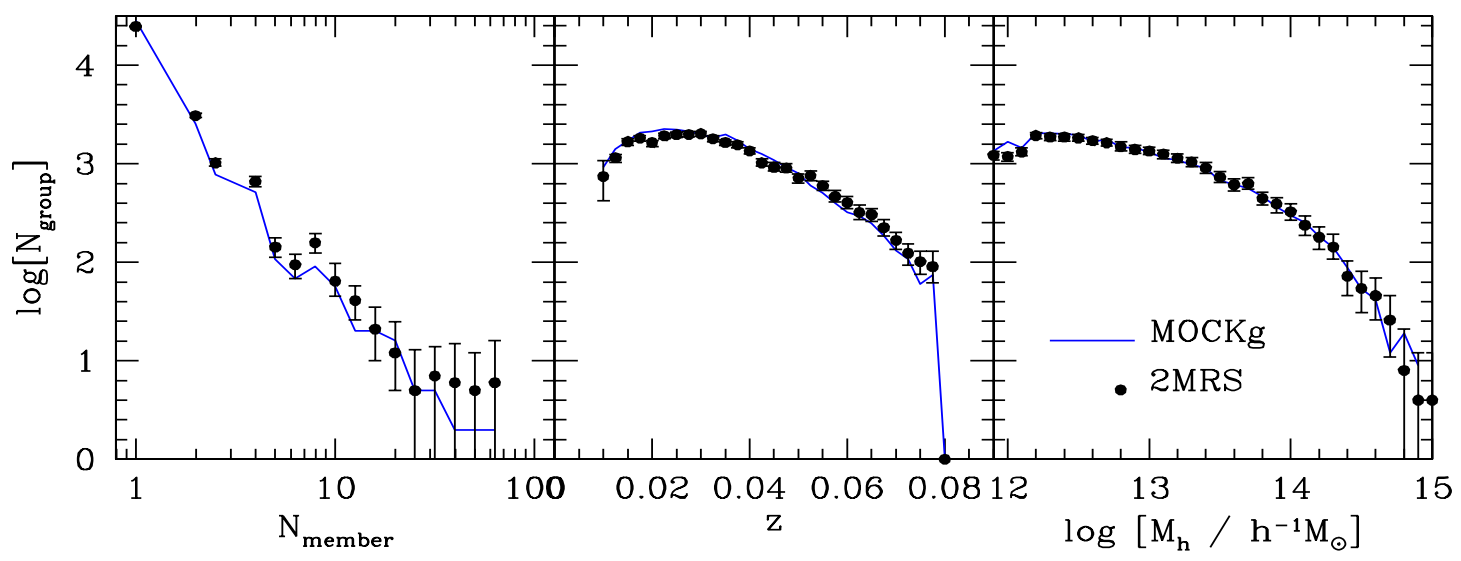

FIG. 11. - The number of groups as function of the number of group members (left panel), group redshift (middle panel) and halo mass (right panel). The solid points with error bars show the results obtained from the $2 \mathrm{MRS}$ group catalog constructed using the modified halo-based group finder. The error bars are given by 1000 bootstrap re-samplings. For comparison, in all panels, we also plot the corresponding distributions obtained from the mock $2 \mathrm{MRS}$ group catalog (MOCKg) using curves.

TABLE 4

COMPARISON BETWEEN HALO MASSES (IN $\left.\log \left[M_{h} / h^{-1} \mathrm{M}_{\odot}\right]\right)$

\begin{tabular}{lccc}
\hline \hline Groups & Lu & T15 & Literature \\
\hline Abell2199 & 14.84 & 15.26 & $14.81^{1}$ \\
Coma & 14.76 & 15.23 & $14.85^{2}$ \\
Abell2634 & 14.59 & 14.90 & $14.61^{3}$ \\
Perseus & 14.59 & 15.07 & \\
Norma & 14.30 & 15.10 & $15.00^{4}$ \\
Virgo & 14.37 & 15.04 & $14.43-14.90^{5}$ \\
Abell1367 & 14.34 & 14.80 & \\
\hline \multicolumn{3}{c}{ NoTE. ${ }^{1}$ Kubo et al. (2009). } & \\
& \\
(2009). & Gavazzi et al. \\
Schindler \& Prieto (1997). ${ }^{4}$ Woudt et al. \\
(2008). ${ }^{5}$ Karachentsev \& Nasonova (2010).
\end{tabular}

the ones obtained form the MOCKg groups are also plotted in this figure. The good agreement between MOCKg and 2MRS indicate that the number distribution of groups as a function of halo mass beyond the redshift completeness $z_{\text {limit }}$ are also quite similar in the MOCKg and 2MRS samples.

\subsection{Comparisons with previous results}

In a recent study, Tully (2015, hereafter T15) identified galaxy groups from the $2 \mathrm{MRS}$ using a modified version of the halo-based group finder developed by Yang et al. (2005a), with halo masses estimated from a scaling relation to the characteristic group luminosity. Tully identified 13,606 groups from a total 24,044 galaxies in the velocity range $3000-$ $10,000 \mathrm{~km} \mathrm{~s}^{-1}$, among which 3,461 have more than one member. In comparison, our group catalog uses a different halo mass estimator and extends to a larger redshift range. In particular, we have used a realistic mock catalog to quantify the reliability of our group finder and the group masses it gives.

To compare with T15, we list in Table. 3 the properties of groups in the redshift range $0.01 \leq z \leq 0.03$, which is comparable to the redshift range used in T15. For a total of 20,921 galaxies, we identified 12,889 groups, which matches well with the results of T15. The richest group has 184 member galaxies in our catalog, which is consistent with 180 member galaxies given by T15. We list the estimated halo masses of some prominent nearby groups in Table. 4, including groups in the Perseus-Pisces filament, Leo cluster, Norma cluster, Virgo and Coma clusters. For comparison, the results given by T15 for the same groups are also listed in the table. In general, the halo masses given by T15 tend to be larger than the masses we obtain. We suspect that this is caused by different definitions of halo masses. To investigate this further, we looked into the literature for the halo masses of the groups in question, and the results are also shown in Table. 4. In general our mass estimates match well with the values given in the literature. The only exception is the Norma cluster, for which our mass estimate is significantly lower. However, Norma is located near the Milky Way Zone of Avoidance, and is severely obscured by the interstellar dust at the optical wavelengths. It is unclear if this is also a significant problem in the near infrared data used here.

We further test our 2MRS group catalogs by comparing with an existing group catalog. This group catalog used here was constructed by Y07 from the New York University ValueAdded Galaxy Catalog (Blanton et al. 2005, NYU-VAGC; ) based on the SDSS Data Release 7. A total of 639,359 galaxies with redshifts $0.01 \leq z \leq 0.20$ and redshift completenesses $C>0.7$ were selected for constructing their group catalog. They found a total of 472,416 groups, among which 23,700 have three or more members. For our comparison, we first cross match the 2MRS galaxies with te SDSS DR7 galaxies according to their coordinates in the sky. With the assumption that galaxies located within $5^{\prime \prime}$ of one another in the sky, and with a redshift difference of $\Delta z<5 \times 10^{-4}$ (corresponding to a velocity of $150 \mathrm{~km} / \mathrm{s}$ ) are the same one, we got a total of 4,528 galaxy pairs, among which 2,938 galaxy pairs are centrals in both group catalogs.

We investigate the estimated halo masses assigned to the same halo in the two galaxy group catalogs. Here halos from the two group catalogs are matched if they have the same central galaxy according to the matched galaxy pairs. Note that, for the SDSS galaxy groups, the halo masses are estimated by the 'RANK' method, which estimates the halo mass of a candidate galaxy group according to its characteristic luminosity, $L_{-19.5}$, defined as the total luminosity of member galaxies brighter than a given luminosity threshold 


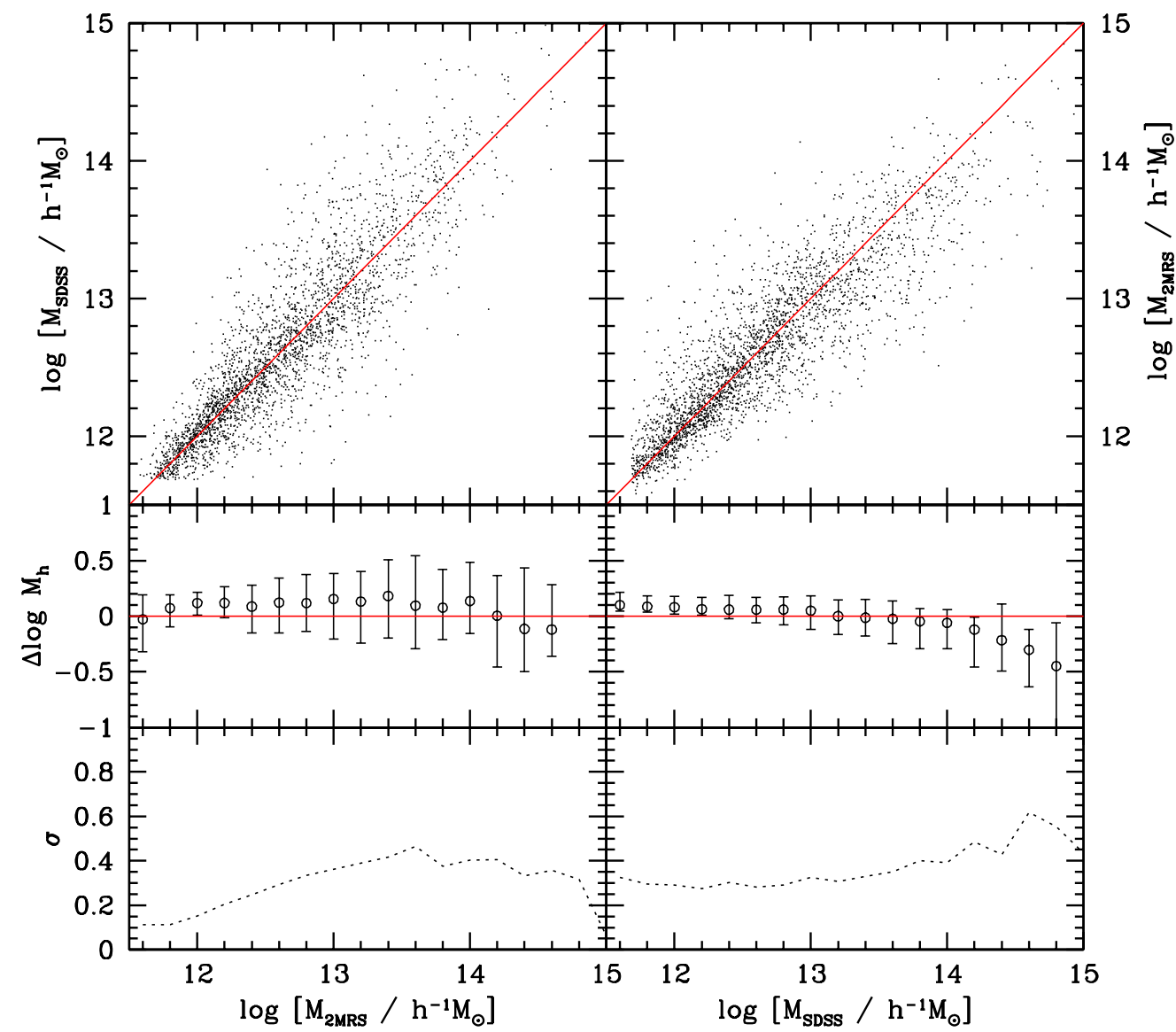

FIG. 12.- Comparison of the estimated halo mass between 2MRS and SDSS DR7 galaxy groups. This is similar to Fig. 8, but with the true halo masses replaced by SDSS DR7 halo masses. The difference between the two halo masses, $\Delta \log M_{h}=\log M_{2 \mathrm{MRS}}-\log M_{\mathrm{SDSS}}$ are also shown in the middle row in bins of halo mass obtained from the 2MRS (left) and SDSS (right), respectively. The error bars indicate the $16 \%-84 \%$ percentiles of the distributions. The standard variation of groups from the redlines in the top two panels are shown as the $\sigma$ curves plotted in the bottom panels.

${ }^{0.1} \mathrm{M}_{r}-5 \log h=-19.5$. The top panels in Fig. 12 show the estimated halo masses for all the 2,938 matched central galaxy pairs given by the $2 \mathrm{MRS}$ and SDSS DR7 group catalogs, respectively. The top left and top right panels plot the same thing, except that the two mass axes are flipped: SDSS mass versus 2 MRS mass in the left and 2 MRS mass versus SDSS mass in the right). Ideally, the two estimated halo masses should be the same, so that all the data points would lie on the red solid line $\left(\log M_{2 \mathrm{MRS}}=\log M_{\mathrm{SDSS}}\right)$. We can see that the two halo masses estimated are tightly correlated with each another, with no obvious systematic bias (see the middle two panels which show the deviations from the perfect line). The typical scatter is $\sim 0$.4dex in medium to massive halo mass range, and $\sim 0.2-0.3$ dex for low mass groups, as shown in the two lower panels. This scatter is roughly consistent with the one shown in Fig. 8 between the group and true halo masses estimated from the mock $2 \mathrm{MRS}$ catalogs.

\section{SUMMARY}

In this paper, we have implemented, tested and applied a modified version of the halo-based group finder developed in Yang et al. (2005a, 2007) to extract galaxy groups from the $2 \mathrm{MRS}$. Covering uniformly about $91 \%$ of the sky, the $2 \mathrm{MRS}$ provides the best available representation of the structures in local universe, and so a group catalog constructed from it is useful for many purposes. However, $2 \mathrm{MRS}$ is quite shallow; in many cases only a few brightest members within a halo can be observed. To deal with this limit, we have updated the halo mass estimate used in the previous group finder with a new method based on 'GAP'. This 'GAP' estimator consists of two parts: (i) a relation between the luminosity of the central galaxy, $L_{c}$, and the halo mass, $M_{h}$, inferred iteratively from abundance matching between the luminosity of central galaxies and the masses of dark matter halos; (2) a luminosity gap correction factor obtained from the luminosity difference between the central galaxy and a faint satellite galaxy.

In order to evaluate the performance of our modified group finder, we have constructed mock $2 \mathrm{MRS}$ galaxy samples based on the observed $K_{s}$-band luminosity function. The group catalog obtained from the mock $2 \mathrm{MRS}$ galaxy catalog shows a $100 \%$ completeness for about $65 \%$ of the most massive groups to $\sim 85 \%$ for groups with halo masses $\log M_{h}<$ $10^{14} h^{-1} \mathrm{M}_{\odot}$. On average, about $80 \%$ of the groups have $80 \%$ completeness. In terms of interlopers, about $65 \%$ of the groups identified have none, and an additional $20 \%$ have an interloper fraction lower than $50 \%$. Further tests on the halo mass estimation show that the deviation of the halo mass between the selected groups and the true halos is $\sim 0.35$ dex over the entire mass range. These tests demonstrate that the modified group finder is reliable for the $2 \mathrm{MRS}$ sample. 
Applying the modified halo-based group finder to the 2MRS, we have obtained a group catalog with a depth to $z \leq 0.08$ and covering $91 \%$ of the whole sky. This $2 \mathrm{MRS}$ group catalog contains a total of 29,904 groups, among which 24,618 are singles and 5,286 have more than one member. Some of the basic properties of the group catalog are presented, including the distributions in richness, in redshift and in halo mass. This catalog provides a useful data base to study galaxies in different environments. In particular, it can be used to reconstruct the mass distribution in the local Universe, as we will do in a forthcoming paper.

\section{ACKNOWLEDGEMENTS}

We thank the anonymous referee for helpful comments that greatly improved the presentation of this paper. This work is supported by 973 Program (No. 2015CB857002), national science foundation of China (grants Nos. 11203054, 11128306, 11121062, 11233005, 11073017), NCET-110879, the Strategic Priority Research Program "The Emergence of Cosmological Structures" of the Chinese Academy of Sciences, Grant No. XDB09000000 and the Shanghai Committee of Science and Technology, China (grant No. 12ZR1452800). We also thank the support of a key laboratory grant from the Office of Science and Technology, Shanghai Municipal Government (No. 11DZ2260700). HJM would like to acknowledge the support of NSF AST-1517528.

A computing facility award on the PI cluster at Shanghai Jiao Tong University is acknowledged. This work is also supported by the High Performance Computing Resource in the Core Facility for Advanced Research Computing at Shanghai Astronomical Observatory.

\section{REFERENCES}

Behroozi, P. S., Conroy, C., \& Wechsler, R. H. 2010, ApJ, 717, 379

Berlind, A. A., \& Weinberg, D. H. 2002, ApJ, 575, 587

Berlind, A. A., Frieman, J., Weinberg, D. H., et al. 2006, ApJS, 167, 1

Blanton, M. R., Schlegel, D. J., Strauss, M. A., et al. 2005, AJ, 129, 2562

Bryan, G. L., Norman, M. L., Stone, J. M., Cen, R., \& Ostriker, J. P. 1995 Computer Physics Communications, 89, 149

Cacciato, M., van den Bosch, F. C., More, S., et al. 2009, MNRAS, 394, 929

Conroy, C., Wechsler, R. H., \& Kravtsov, A. V. 2006, ApJ, 647, 201

Cooray, Asantha, 2006, MNRAS, 365, 842C

Crook, A. C., Huchra, J. P., Martimbeau, N., et al. 2007, ApJ, 655, 790

Croton, D. J., Springel, V., White, S. D. M., et al. 2006, MNRAS, 365, 11

Davis, M., Efstathiou, G., Frenk, C. S., \& White, S. D. M. 1985, ApJ, 292, 371

Eke, V. R., Baugh, C. M., Cole, S., et al. 2004, MNRAS, 348, 866

Erfanianfar, G., Popesso, P., Finoguenov, A., et al. 2014, MNRAS, 445, 2725

Felten, J. E. 1976, ApJ, 207, 700

Gavazzi, R., Adami, C., Durret, F., et al. 2009, A\&A, 498, L33

Guo, Q., White, S., Li, C., \& Boylan-Kolchin, M. 2010, MNRAS, 404, 1111

Hinshaw, G., Larson, D., Komatsu, E., et al. 2013, ApJS, 208, 19

Huchra, J. P., \& Geller, M. J. 1982, ApJ, 257, 423

Huchra, J. P., Macri, L. M., Masters, K. L., et al. 2012, ApJS, 199, 26

Jarrett, T. H., Chester, T., Cutri, R., et al. 2000, AJ, 119, 2498

Jarrett, T. H., Chester, T., Cutri, R., Schneider, S. E., \& Huchra, J. P. 2003, AJ, 125,525

Jiang, N., Wang, H., Mo, H., et al. 2016, arXiv:1602.08825

Jing, Y. P., Mo, H. J., \& Börner, G. 1998, ApJ, 494, 1

Kang, X., Jing, Y. P., Mo, H. J., Borner, G. 2005, ApJ, 631, 21

Karachentsev, I. D., Tully, R. B., Wu, P.-F., Shaya, E. J., \& Dolphin, A. E. 2014, ApJ, 782, 4

Karachentsev, I. D., Makarov, D. I., \& Kaisina, E. I. 2013, AJ, 145, 101

Karachentsev, I. D., \& Nasonova, O. G. 2010, MNRAS, 405, 1075

Karachentsev, I. D., \& Makarov, D. A. 1996, AJ, 111, 794

Kim, R. S. J., Kepner, J. V., Postman, M., et al. 2002, AJ, 123, 20

Koester, B. P., McKay, T. A., Annis, J., et al. 2007, ApJ, 660, 221

Kravtsov, A. V., Klypin, A., \& Hoffman, Y. 2002, ApJ, 571, 563

Kubo, J. M., Annis, J., Hardin, F. M., et al. 2009, ApJ, 702, L110

Lan, T.-W., Ménard, B., \& Mo, H. 2016, MNRAS, 459, 3998

Li, S.-J., Zhang, Y.-C., Yang, X.-H., et al. 2016, Research in Astronomy and Astrophysics, 16, 013

Lu, Z., Mo, H. J., Lu, Y., et al. 2015, MNRAS, 450, 1604

Lu, Z., Mo, H. J., Lu, Y., et al. 2014, MNRAS, 439, 1294

Lu, Y., Yang, X., \& Shen, S. 2015, ApJ, 804, 55

Mo, H. J., \& White, S. D. M. 2002, MNRAS, 336, 112

Mo, H. J., Mao, S., \& White, S. D. M. 1999, MNRAS, 304, 175

Navarro, J. F., Frenk, C. S., \& White, S. D. M. 1997, ApJ, 490, 493

Nurmi, P., Heinämäki, P., Sepp, T., et al. 2013, MNRAS, 436, 380

Old, L., Skibba, R. A., Pearce, F. R., et al. 2014, MNRAS, 441, 1513

Old, L., Wojtak, R., Mamon, G. A., et al. 2015, MNRAS, 449, 1897
Peacock, J.A \& Smith, R. E, 2000, MNRAS, 318, 1144P

Rodríguez-Puebla, A., Avila-Reese, V., Yang, X., et al. 2015, ApJ, 799, 130 Schechter, P. 1976, ApJ, 203, 297

Schmidt, M. 1968, ApJ, 151, 393

Schindler, S., \& Prieto, M. A. 1997, A\&A, 327, 37

Springel, V., White, S. D. M., Jenkins, A., et al. 2005, Nature, 435, 629

Springel, V. 2010, MNRAS, 401, 791

Tago, E., Saar, E., Tempel, E., et al. 2010, A\&A, 514, AA102

Tal, T., Dekel, A., Oesch, P., et al. 2014, ApJ, 789, 164

Tempel, E., Kipper, R., Tamm, A., et al. 2016, A\&A, 588, A14

Teyssier, R. 2002, A\&A, 385, 337

Tinker, J. L. 2005, Ph.D. Thesis, 3180

Tinker, J., Kravtsov, A. V., Klypin, A., et al. 2008, ApJ, 688, 709-728

Trujillo-Gomez, S., Klypin, A., Primack, J., \& Romanowsky, A. J. 2011, ApJ, 742, 16

Tully, R. B. 2015, AJ, 149, 171

Vale, A., \& Ostriker, J. P. 2004, MNRAS, 353, 189

Vale, A., \& Ostriker, J. P. 2006, MNRAS, 371, 1173

van den Bosch, F. C. 2002, MNRAS, 332, 456

van den Bosch, F. C., Yang, X., \& Mo, H. J. 2003, MNRAS, 340, 771

van den Bosch, F. C., Yang, X., Mo, H. J., et al. 2007, MNRAS, 376, 841

Wadsley, J. W., Stadel, J., \& Quinn, T. 2004, New Astronomy, 9, 137

Wang, H., Mo, H. J., Yang, X., \& van den Bosch, F. C. 2012, MNRAS, 420, 1809

Wang, H., Mo, H. J., Yang, X., \& van den Bosch, F. C. 2013, ApJ, 772, 63

Wang, H., Mo, H. J., Yang, X., Jing, Y. P., \& Lin, W. P. 2014, ApJ, 794, 94

Weinmann, S. M., van den Bosch, F. C., Yang, X., \& Mo, H. J. 2006a,

MNRAS, 366, 2

Weinmann, S. M., van den Bosch, F. C., Yang, X., et al. 2006b, MNRAS, 372,1161

Woudt, P. A., Kraan-Korteweg, R. C., Lucey, J., Fairall, A. P., \& Moore, S. A. W. 2008, MNRAS, 383, 445

Yan, R., Madgwick, D. S., \& White, M. 2003, ApJ, 598, 848

Yang, X., Mo, H. J., van den Bosch, F. C., 2003, MNRAS, 339, 1057Y

Yang, X., Mo, H. J., Jing, Y. P., van den Bosch, F. C., \& Chu, Y. 2004, MNRAS, 350, 1153

Yang, X., Mo, H. J., van den Bosch, F. C., \& Jing, Y. P. 2005a, MNRAS, 356,1293

Yang, X., Mo, H. J., Jing, Y. P., \& van den Bosch, F. C. 2005b, MNRAS, 358,217

Yang, X., Mo, H. J., van den Bosch, F. C., et al. 2005c, MNRAS, 362, 711

Yang, X., Mo, H. J., van den Bosch, F. C., et al. 2007, ApJ, 671, 153

Yang, X., Mo, H. J., \& van den Bosch, F. C. 2008, ApJ, 676, 248

Yang, X., Mo, H. J., van den Bosch, F. C., Zhang, Y., \& Han, J. 2012, ApJ, 752,41

Zhao, D. H., Jing, Y. P., Mo, H. J., Börner, G. 2009, ApJ, 707, 354

Zheng, Z., Berlind, A. A., Weinberg, D. H., et al. 2005, ApJ, 633, 791 